\title{
TIME FINITE ELEMENT METHODS FOR STRUCTURAL DYNAMICS
}

\author{
GREGORY M. HULBERT* \\ Department of Mechanical Engineering and Applied Mechanics, 321 W. E. Lay Automotive Lab, The University of \\ Michigan, Ann Arbor, Michigan 48109-2121, U.S.A.
}

\begin{abstract}
SUMMARY
Time finite element methods are developed for the equations of structural dynamics. The approach employs the time-discontinuous Galerkin method and incorporates stabilizing terms having least-squares form. These enable a general convergence theorem to be proved in a norm stronger than the energy norm. Results are presented from finite difference analyses of the time-discontinuous Galerkin and least-squares methods with various temporal interpolations and commonly used finite difference methods for structural dynamics. These results show that, for particular interpolations, the time finite element method exhibits improved accuracy and stability.
\end{abstract}

\section{INTRODUCTION}

One approach towards formulating algorithms for solving partial differential equations associated with time-dependent phenomena is to first discretize the spatial domain of the problem using typical finite element techniques. This results in a system of ordinary differential equations with time as the independent variable. Most commonly used transient dynamics algorithms are derived by discretizing the ordinary differential equations using traditional finite difference methods. Alternatively, the ordinary differential equations may be discretized using finite elements in time. For example, in Argyris and Scharpf, ${ }^{1}$ Bazzi and Anderheggen, ${ }^{2} \mathrm{Hoff}$ and Pahl, ${ }^{3,4} \mathrm{Hoff}$ et al., ${ }^{5}$ Kawahara and Hasegawa, ${ }^{6}$ Kujawski and Desai ${ }^{7}$ and Zienkiewicz and co-workers, ${ }^{8-12}$ continuous functions in time are substituted into the ordinary differential equations emanating from semidiscretizations, multiplied by weighting functions and integrated over time intervals. Many traditional ordinary differential equation algorithms were rederived in this manner as well as useful new algorithms for structural dynamics.

Another approach to discretizing the temporal domain is to permit the unknown fields to be discontinuous with respect to time. The time-discontinuous Galerkin method was originally developed for first-order hyperbolic equations by Reed and Hill ${ }^{13}$ and Lesaint and Raviart. ${ }^{14}$ This method has been successfully applied to problems in incompressible and compressible fluid dynamics, heat conduction and elastodynamics; see References 15-29.

The time-discontinuous Galerkin method leads to stable, higher-order accurate finite element methods. It was first shown in Delfour et al., ${ }^{16}$ Johnson $^{30}$ and Lesaint and Raviart ${ }^{14}$ that the time-discontinuous Galerkin method leads to $A$-stable, higher-order accurate ordinary differential equation solvers. Furthermore, the time-discontinuous framework seems conducive to the

\footnotetext{
*Assistant Professor of Mechanical Engineering and Applied Mechanics
}

0029-5981/92/020307-25\$12.50

(C) 1992 by John Wiley \& Sons, Ltd.

Received 12 September 1990

Revised 24 January 1991 
establishment of rigorous convergence proofs and error estimates; see, e.g. References 17-20, 22, $23,27,30-39$.

This paper extends the work presented in Hughes and Hulbert ${ }^{18}$ and Hulbert and Hughes ${ }^{20}$ to structural dynamics. We show that the time finite element method generates a family of unconditionally stable higher-order accurate algorithms for solving systems of second-order ordinary differential equations associated with structural dynamics. An outline of the paper follows. After presenting the equations of structural dynamics, two general formulations of the time finite element method are presented. In the single-field formulation, presented in Section 2, displacement is chosen as the unknown field, while in the $t w o-f i e l d$ formulation, given in Section 3, displacement and velocity are the unknown fields. Stability and convergence are proved for both formulations. In Section 4, the single-field and two-field formulations are compared. Results are presented in Section 5 from finite difference analyses of the time finite element methods using various combinations of interpolations. These results are compared to those obtained for several commonly used time integration algorithms for structural dynamics. Conclusions are drawn in Section 6.

The ordinary differential equations associated with the semidiscrete form of linear elastodynamics have the form

$$
\begin{aligned}
\mathbf{M u ̈}+C \dot{\mathbf{u}}+\mathbf{K u} & =\mathbf{F} \\
\dot{\mathbf{u}}_{0} & =\mathbf{v}_{0} \\
\mathbf{u}_{0} & =\mathbf{d}_{0}
\end{aligned}
$$

where $\mathbf{M}, \mathbf{C}$ and $\mathbf{K}$ are the respective mass, damping and stiffness matrices; $\mathbf{u}=\mathbf{u}(t)$ is the vector of unknown nodal displacements having dimension $n_{\mathrm{eq}} ; \mathbf{F}$ is the prescribed load vector; $\mathbf{v}_{0}$ and $\mathbf{d}_{0}$ are the initial velocity and displacement vectors, respectively. A superposed dot denotes differentiation with respect to time. $\mathbf{M}$ is assumed to be symmetric positive-definite while $\mathbf{C}$ and $\mathbf{K}$ are assumed to be symmetric positive-semidefinite.

\section{A SINGLE-FIELD TIME FINITE ELEMENT FORMULATION}

\subsection{Variational equations}

Consider a partition of the time domain, $I=] 0, T\left[\right.$, having the form $0=t_{0}<t_{1}<\ldots<t_{N}$ $=T$. Let $\left.I_{n}=\right] t_{n-1}, t_{n}\left[\right.$ and $\Delta t_{n}=t_{n}-t_{n-1}$. Assuming the function $\mathrm{w}(t)$ to be discontinuous at time $t_{n}$, the temporal jump operator is defined by

$$
\llbracket \mathbf{w}\left(t_{n}\right) \rrbracket=\mathbf{w}\left(t_{n}^{+}\right)-\mathbf{w}\left(t_{n}^{-}\right)
$$

where

$$
\mathbf{w}\left(t_{n}^{ \pm}\right)=\lim _{\varepsilon \rightarrow 0 \pm} \mathbf{w}\left(t_{n}+\varepsilon\right)
$$

The following notation is used to simplify the subsequent exposition:

$$
(\mathrm{w}, \mathrm{u})_{I_{n}}=\int_{I_{n}} \mathrm{w} \cdot \mathrm{ud} t
$$

Let $\mathscr{P}^{k}$ denote the space of $k$ th order polynomials. The finite element interpolation functions for the trial displacements are

$$
\mathscr{S}^{h}=\left\{\mathbf{u}^{h} \in \bigcup_{n=1}^{N}\left(\mathscr{P}^{k}\left(I_{n}\right)\right)^{n_{\mathrm{eq}}}\right\}
$$


By construction, the interpolation functions are continuous within each time interval and may be discontinuous across the time intervals. Since the initial conditions are weakly enforced, the displacement weighting function space is identical to the trial displacement space.

The statement of the time-discontinuous Galerkin method for the single-field formulation is: Find $\mathbf{u}^{h} \in \mathscr{S}^{h}$ such that for all $\mathbf{w}^{h} \in \mathscr{S}^{h}$,

$$
b_{\mathrm{DG}}\left(\mathbf{w}^{h}, \mathbf{u}^{h}\right)_{n}=l_{\mathrm{DG}}\left(\mathbf{w}^{h}\right)_{n}, \quad n=1,2, \ldots, N
$$

where

$$
\begin{aligned}
b_{\mathrm{DG}}\left(\mathbf{w}^{h}, \mathbf{u}^{h}\right)_{n}= & \left(\dot{\mathbf{w}}^{h}, \mathscr{L} \mathbf{u}^{h}\right)_{I_{n}} \\
& +\dot{\mathbf{w}}^{h}\left(t_{n-1}^{+}\right) \cdot \mathbf{M} \dot{\mathbf{u}}^{h}\left(t_{n-1}^{+}\right) \\
& +\mathbf{w}^{h}\left(t_{n-1}^{+}\right) \cdot \mathbf{K} \mathbf{u}^{h}\left(t_{n-1}^{+}\right) \\
l_{\mathrm{DG}}\left(\mathbf{w}^{h}\right)_{n}= & \left(\dot{\mathbf{w}}^{h}, \mathbf{F}\right)_{I_{n}} \\
& +\dot{\mathbf{w}}^{h}\left(t_{n-1}^{+}\right) \cdot \mathbf{M u}^{h}\left(t_{n-1}^{-}\right) \\
& +\mathbf{w}^{h}\left(t_{n-1}^{+}\right) \cdot \mathbf{K u}^{h}\left(t_{n-1}^{-}\right), \quad n=2,3, \ldots, N \\
l_{\mathrm{DG}}\left(\mathbf{w}^{h}\right)_{1}= & \left(\dot{\mathbf{w}}^{h}, \mathbf{F}\right)_{I_{1}} \\
& +\dot{\mathbf{w}}^{h}\left(0^{+}\right) \cdot \mathbf{M} \mathbf{v}_{0}+\mathbf{w}^{h}\left(0^{+}\right) \cdot \mathbf{K d}_{0}
\end{aligned}
$$

and

$$
\mathscr{L} \mathbf{u}^{h}=\mathbf{M} \ddot{\mathbf{u}}^{h}+\mathbf{C} \dot{\mathbf{u}}^{h}+\mathbf{K} \mathbf{u}^{h}
$$

Note $l_{\mathrm{DG}}\left(\mathbf{w}^{h}\right)_{1}$ is defined from the general expression for $l_{\mathrm{DG}}\left(\mathbf{w}^{h}\right)_{n}$ by replacing $\dot{\mathbf{u}}^{h}\left(t_{n-1}^{-}\right)$and $\mathbf{u}^{h}\left(t_{n-1}^{-}\right)$by the initial conditions $\mathbf{v}_{0}$ and $\mathbf{d}_{0}$, respectively. The last two terms of (9), in combination with the last two terms of $(10)$, weakly enforce the initial conditions for each time interval. As will be seen in the stability analysis, these jump terms are stabilizing operators and have the effect of 'upwinding' information with respect to time.

While stability is easily proved for the time-discontinuous Galerkin method, convergence, as measured in the $\|\mid\| \cdot \|$-norm defined in Section 2.2 , has been proved only for $\mathscr{P}{ }^{1}$ polynomials, that is, for linear elements in time. The time-discontinuous Galerkin/least-squares method was developed to circumvent this limitation.

The statement of the time-discontinuous Galerkin/least-squares method for the single-field formulation is:

Find $\mathbf{u}^{h} \in \mathscr{S}^{h}$ such that for all $\mathbf{w}^{h} \in \mathscr{S}^{h}$,

$$
b_{\mathrm{GLS}}\left(\mathbf{w}^{h}, \mathbf{u}^{h}\right)_{n}=l_{\mathrm{GLS}}\left(\mathbf{w}^{h}\right)_{n}, \quad n=1,2, \ldots, N
$$

where

$$
\begin{gathered}
b_{\mathrm{GLS}}\left(\mathbf{w}^{h}, \mathbf{u}^{h}\right)_{n}=b_{\mathrm{DG}}\left(\mathbf{w}^{h}, \mathbf{u}^{h}\right)_{n}+\left(\mathscr{L} \mathbf{w}^{h}, \tau \mathbf{M}^{-1} \mathscr{L} \mathbf{u}^{h}\right)_{I_{n}} \\
l_{\mathrm{GLS}}\left(\mathbf{w}^{h}\right)_{n}=l_{\mathrm{DG}}\left(\mathbf{w}^{h}\right)_{n}+\left(\mathscr{L} \mathbf{w}^{h}, \tau \mathbf{M}^{-1} \mathbf{F}\right)_{I_{n}}
\end{gathered}
$$

The least-squares contribution in (14) adds sufficient stability to prove convergence for polynomials of arbitrary-order while maintaining the accuracy of the underlying time-discontinuous Galerkin method. The scalar parameter, $\tau$, has dimensions of time and is referred to as the intrinsic time-scale. 


\subsection{Stability and convergence analyses}

A sufficiently smooth exact solution of (1)-(3), u, satisfies

$$
b_{\mathrm{GLS}}\left(\mathbf{w}^{h}, \mathbf{u}\right)_{n}=l_{\mathrm{GLS}}\left(\mathbf{w}^{h}\right)_{n}
$$

for all $w^{h} \in \mathscr{S}^{h}$ and $n=1,2, \ldots, N$. This can be easily seen from the Euler-Lagrange form of (13):

$$
\begin{aligned}
0= & b_{\mathrm{GLS}}\left(\mathbf{w}^{h}, \mathbf{u}^{h}\right)_{n}-l_{\mathrm{GLS}}\left(\mathbf{w}^{h}\right)_{n} \\
= & \left(\dot{\mathbf{w}}^{h}+\tau \mathbf{M}^{-1} \mathscr{L} \mathbf{w}^{h}, \mathscr{L} \mathbf{u}^{h}-\mathbf{F}\right)_{I_{n}} \\
& +\dot{\mathbf{w}}^{h}\left(t_{n-1}^{+}\right) \cdot \mathbf{M} \llbracket \dot{\mathbf{u}}^{h}\left(t_{n-1}\right) \rrbracket \\
& \left.+\mathbf{w}^{h}\left(t_{n-1}^{+}\right) \cdot \mathbf{K} \llbracket \mathbf{u}^{h}\left(t_{n-1}\right)\right]
\end{aligned}
$$

The Euler-Lagrange form illustrates weak enforcement of displacement and velocity continuity at the beginning of each time interval.

For purposes of analysis, it is convenient to sum (13) over the time intervals and after rearranging the terms,

where

$$
b_{\mathrm{GLS}}\left(\mathbf{w}^{h}, \mathbf{u}^{h}\right)=l_{\mathrm{GLS}}\left(\mathbf{w}^{h}\right)
$$

$$
\begin{aligned}
b_{\mathrm{GLS}}\left(\mathbf{w}^{h}, \mathbf{u}^{h}\right)= & \sum_{n=1}^{N}\left\{\left(\dot{\mathbf{w}}^{h}, \mathscr{L} \mathbf{u}^{h}\right)_{I_{n}}+\left(\mathscr{L} \mathbf{w}^{h}, \tau \mathbf{M}^{-1} \mathscr{L} \mathbf{u}^{h}\right)_{I_{n}}\right\} \\
& \left.\left.+\sum_{n=1}^{N-1}\left\{\dot{\mathbf{w}}^{h}\left(t_{n}^{+}\right) \cdot \mathbf{M} \llbracket \dot{\mathbf{u}}^{h}\left(t_{n}\right)\right]+\mathbf{w}^{h}\left(t_{n}^{+}\right) \cdot \mathbf{K} \llbracket \mathbf{u}^{h}\left(t_{n}\right)\right]\right\} \\
& +\dot{\mathbf{w}}^{h}\left(0^{+}\right) \cdot \mathbf{M} \dot{\mathbf{u}}^{h}\left(0^{+}\right)+\mathbf{w}^{h}\left(0^{+}\right) \cdot \mathbf{K} \mathbf{u}^{h}\left(0^{+}\right) \\
l_{\mathrm{GLS}}\left(\mathbf{w}^{h}\right)= & \sum_{n=1}^{N}\left\{\left(\dot{\mathbf{w}}^{h}, \mathbf{F}\right)_{I_{n}}+\left(\mathscr{L} \mathbf{w}^{h}, \tau \mathbf{M}^{-1} \mathbf{F}\right)_{I_{n}}\right\} \\
& +\dot{w}^{h}\left(0^{+}\right) \cdot \mathbf{M} \mathbf{v}_{0}+\mathbf{w}^{h}\left(0^{+}\right) \cdot \mathbf{K d} \mathbf{d}_{0}
\end{aligned}
$$

By (16), (18) is also satisfied by substituting a sufficiently smooth exact solution, $u$, for $\mathbf{u}^{h}$. Thus, for all $w^{h} \in \mathscr{S}^{h}$,

where

$$
b_{\mathrm{GLS}}\left(\mathbf{w}^{h}, \mathbf{e}\right)=0
$$

$$
\mathbf{e}=\mathbf{u}^{h}-\mathbf{u}
$$

This is the consistency condition. Clearly the time-discontinuous Galerkin formulation $(\tau=0)$ also satisfies a consistency condition.

2.2.1. Stability. A natural measure of stability for (1)-(3) is the total energy, which, for the single-field formulation, is given by

$$
\mathscr{E}\left(\mathbf{w}^{h}\right)=\frac{1}{2} \dot{\mathbf{w}}^{h} \cdot \mathbf{M} \dot{\mathbf{w}}^{h}+\frac{1}{2} \mathbf{w}^{h} \cdot \mathbf{K} \mathbf{w}^{h}
$$

The norm associated with (18) and in which stability and convergence are proved is defined by

$$
\begin{aligned}
\left\|w^{h}\right\| \|^{2}= & \mathscr{E}\left(\mathbf{w}^{h}\left(T^{-}\right)\right)+\mathscr{E}\left(\mathbf{w}^{h}\left(0^{+}\right)\right)+\sum_{n=1}^{N-1} \mathscr{E}\left(\llbracket \mathbf{w}^{h}\left(t_{n}\right) \rrbracket\right) \\
& +\sum_{n=1}^{N}\left\{\left(\dot{w}^{h}, \mathbf{C} \dot{w}^{h}\right)_{I_{n}}+\left(\mathscr{L} \mathbf{w}^{h}, \tau \mathbf{M}^{-1} \mathscr{L} \mathbf{w}^{h}\right)_{I_{n}}\right\}
\end{aligned}
$$


Lemma 1

$$
b_{\mathrm{GLS}}\left(\mathbf{w}^{h}, \mathbf{w}^{h}\right)=\left\|\mathbf{w}^{h}\right\|^{2}
$$

This is the stability condition. To prove (25), we need to prove a preliminary result, namely:

Lemma 2

$$
\begin{aligned}
b_{\mathrm{DG}}\left(\mathbf{w}^{h}, \mathbf{w}^{h}\right)= & \mathscr{E}\left(\mathbf{w}^{h}\left(T^{-}\right)\right)+\mathscr{E}\left(\mathbf{w}^{h}\left(0^{+}\right)\right)+\sum_{n=1}^{N-1} \mathscr{E}\left(\left[\mathbf{w}^{h}\left(t_{n}\right)\right]\right) \\
& +\sum_{n=1}^{N}\left(\dot{\mathbf{w}}^{h}, \mathbf{C} \dot{\mathbf{w}}^{h}\right)_{I_{n}}
\end{aligned}
$$

Proof

$$
\begin{aligned}
\sum_{n=1}^{N}\left(\dot{\mathbf{w}}^{h}, \mathbf{M} \ddot{\mathbf{w}}^{h}\right)_{I_{n}}= & \sum_{n=1}^{N} \int_{t_{n-1}}^{t_{n}} \dot{\mathbf{w}}^{h} \cdot \mathbf{M} \ddot{\mathbf{w}}^{h} \mathrm{~d} t \\
= & \sum_{n=1}^{N} \int_{t_{n-1}}^{t_{n}} \frac{1}{2} \frac{\mathrm{d}}{\mathrm{d} t}\left(\dot{\mathbf{w}}^{h} \cdot \mathbf{M} \dot{\mathbf{w}}^{h}\right) \mathrm{d} t \\
= & \frac{1}{2} \dot{\mathbf{w}}^{h}\left(T^{-}\right) \cdot \mathbf{M} \dot{\mathbf{w}}^{h}\left(T^{-}\right)-\frac{1}{2} \dot{\mathbf{w}}^{h}\left(0^{+}\right) \cdot \mathbf{M} \dot{\mathbf{w}}^{h}\left(0^{+}\right) \\
& +\frac{1}{2} \sum_{n=1}^{N-1}\left\{\dot{\mathbf{w}}^{h}\left(t_{n}^{-}\right) \cdot \mathbf{M} \dot{\mathbf{w}}^{h}\left(t_{n}^{-}\right)-\dot{\mathbf{w}}^{h}\left(t_{n}^{+}\right) \cdot \mathbf{M} \dot{\mathbf{w}}^{h}\left(t_{n}^{+}\right)\right\}
\end{aligned}
$$

Therefore,

$$
\begin{aligned}
\sum_{n=1}^{N}\left(\dot{\mathbf{w}}^{h}, \mathbf{M} \ddot{\mathbf{w}}^{h}\right)_{I_{n}}+\sum_{n=1}^{N-1} \dot{\mathbf{w}}^{h}\left(t_{n}^{+}\right) \cdot \mathbf{M}\left[\dot{\mathbf{w}}^{h}\left(t_{n}\right)\right]+\dot{\mathbf{w}}^{h}\left(0^{+}\right) \cdot \mathbf{M} \dot{\mathbf{w}}^{h}\left(0^{+}\right) \\
=\frac{1}{2} \dot{\mathbf{w}}^{h}\left(T^{-}\right) \cdot \mathbf{M} \dot{\mathbf{w}}^{h}\left(T^{-}\right)+\frac{1}{2} \dot{\mathbf{w}}^{h}\left(0^{+}\right) \cdot \mathbf{M} \dot{\mathbf{w}}^{h}\left(0^{+}\right) \\
\quad+\sum_{n=1}^{N-1}\left\{\frac{1}{2} \dot{\mathbf{w}}^{h}\left(t_{n}^{-}\right) \cdot \mathbf{M} \dot{\mathbf{w}}^{h}\left(t_{n}^{-}\right)-\frac{1}{2} \dot{\mathbf{w}}^{h}\left(t_{n}^{+}\right) \cdot \mathbf{M} \dot{\mathbf{w}}^{h}\left(t_{n}^{+}\right)\right. \\
\left.\quad+\dot{\mathbf{w}}^{h}\left(t_{n}^{+}\right) \cdot \mathbf{M} \dot{\mathbf{w}}^{h}\left(t_{n}^{+}\right)-\dot{\mathbf{w}}^{h}\left(t_{n}^{+}\right) \cdot \mathbf{M} \dot{\mathbf{w}}^{h}\left(t_{n}^{-}\right)\right\} \\
=\frac{1}{2} \dot{\mathbf{w}}^{h}\left(T^{-}\right) \cdot \mathbf{M} \dot{\mathbf{w}}^{h}\left(T^{-}\right)+\frac{1}{2} \dot{\mathbf{w}}^{h}\left(0^{+}\right) \cdot \mathbf{M} \dot{\mathbf{w}}^{h}\left(0^{+}\right) \\
\quad+\frac{1}{2} \sum_{n=1}^{N-1}\left[\dot{\mathbf{w}}^{h}\left(t_{n}\right)\right] \cdot \mathbf{M}\left[\dot{\mathbf{w}}^{h}\left(t_{n}\right)\right]
\end{aligned}
$$

Similarly,

$$
\begin{aligned}
& \left.\sum_{n=1}^{N}\left(\dot{\mathbf{w}}^{h}, \mathbf{K} \mathbf{w}^{h}\right)_{I_{n}}+\sum_{n=1}^{N-1} \mathbf{w}^{h}\left(t_{n}^{+}\right) \cdot \mathbf{K} \llbracket \mathbf{u}^{h}\left(t_{n}\right)\right]+\mathbf{w}^{h}\left(0^{+}\right) \cdot \mathbf{K} \mathbf{w}^{h}\left(0^{+}\right) \\
= & \frac{1}{2} \mathbf{w}^{h}\left(T^{-}\right) \cdot \mathbf{K} \mathbf{w}^{h}\left(T^{-}\right)+\frac{1}{2} \mathbf{w}^{h}\left(0^{+}\right) \cdot \mathbf{K} \mathbf{w}^{h}\left(0^{+}\right) \\
& +\frac{1}{2} \sum_{n=1}^{N-1} \llbracket \mathbf{w}^{h}\left(t_{n}\right) \rrbracket \cdot \mathbf{K} \llbracket \mathbf{w}^{h}\left(t_{n}\right) \rrbracket
\end{aligned}
$$


Combining (28) and (29) and using (19) (with $\tau=0$ ) and (23) completes the proof.

Lemma 1 follows directly from Lemma 2 using (19) and (24).

Theorem 1 (Energy decay inequality). In the absence of external forces, i.e. $\mathbf{F}=\mathbf{0}$, the total energy at the end of a time interval, e.g. $\mathscr{E}\left(\mathbf{u}^{h}\left(t_{n}^{-}\right)\right)$, computed using the Galerkin/least-squares method, (13), is bounded by the initial total energy.

Proof. With $\mathbf{F}=\mathbf{0}$,

$$
\begin{aligned}
l_{\mathrm{GLS}}\left(\mathbf{u}^{h}\right)= & \dot{\mathbf{u}}^{h}\left(0^{+}\right) \cdot \mathbf{M} \mathbf{v}_{0}+\mathbf{u}^{h}\left(0^{+}\right) \cdot \mathbf{K} \mathbf{d}_{0} \\
\leqslant & \left|\dot{\mathbf{u}}^{h}\left(0^{+}\right) \cdot \mathbf{M} \mathbf{v}_{0}+\mathbf{u}^{h}\left(0^{+}\right) \cdot \mathbf{K} \mathbf{d}_{0}\right| \\
\leqslant & \frac{1}{2} \dot{\mathbf{u}}^{h}\left(0^{+}\right) \cdot \mathbf{M} \dot{\mathbf{u}}^{h}\left(0^{+}\right)+\frac{1}{2} \mathbf{v}_{0} \cdot \mathbf{M} \mathbf{v}_{0} \\
& +\frac{1}{2} \mathbf{u}^{h}\left(0^{+}\right) \cdot \mathbf{K} \mathbf{u}^{h}\left(0^{+}\right)+\frac{1}{2} \mathbf{d}_{0} \cdot \mathbf{K} \mathbf{d}_{0} \\
= & \mathscr{E}\left(\mathbf{u}^{h}\left(0^{+}\right)\right)+\mathscr{E}\left(\mathbf{u}_{0}\right)
\end{aligned}
$$

where $\mathscr{E}\left(\mathbf{u}_{0}\right)$ denotes the initial total energy. Using (18), Lemma 1, (30), and the definition of $\left\|\left|\mathbf{w}^{h} \|\right|^{2}\right.$,

$$
\mathscr{E}\left(\mathbf{u}^{h}\left(T^{-}\right)\right) \leqslant \mathscr{E}\left(\mathbf{u}_{0}\right)
$$

Since $T$ is arbitrary,

$$
\mathscr{E}\left(\mathbf{u}^{h}\left(t_{n}^{-}\right)\right) \leqslant \mathscr{E}\left(\mathbf{u}_{0}\right), \quad n=1,2, \ldots, N
$$

Theorem 1 also holds for the time-discontinuous Galerkin method $(\tau=0)$.

2.2.2. Convergence. Let $\Pi u \in \mathscr{S}^{h}$ denote an interpolant of $\mathbf{u}$. The error, e, can be written as

$$
\mathbf{e}=\mathbf{e}^{h}+\boldsymbol{\eta}
$$

where

$$
\begin{aligned}
\mathbf{e}^{h} & =\left(\mathbf{u}^{h}-\Pi \mathbf{u}\right) \in \mathscr{S}^{h} \\
\boldsymbol{\eta} & =\Pi \mathbf{u}-\mathbf{u}
\end{aligned}
$$

Theorem 2 (Error estimates). Assume $\tau$ satisfies

$$
c_{1} \Delta t \leqslant \tau \leqslant c_{2} \Delta t
$$

where $c_{1}$ and $c_{2}$ are positive constants $\left(c_{2}>c_{1}\right)$ and

$$
\Delta t=\max _{n=1,2, \ldots, N} \Delta t_{n}
$$

Let $H^{k+1}(I)$ denote the Sobolev space of functions that possesses square-integrable generalized derivatives of order $k+1$. Assuming $\mathbf{u} \in\left(H^{k+1}(I)\right)^{n_{e q}}$, then the interpolation error, $\boldsymbol{\eta}$, satisfies:

$$
\begin{gathered}
\sum_{n=1}^{N}(\dot{\boldsymbol{\eta}}, \mathbf{C} \dot{\boldsymbol{\eta}})_{I_{n}} \leqslant c(\mathbf{u}) \Delta t^{2 k} \\
\sum_{n=1}^{N}\left(\dot{\boldsymbol{\eta}}, \tau^{-1} \mathbf{M} \dot{\boldsymbol{\eta}}\right)_{I_{n}} \leqslant c(\mathbf{u}) \Delta t^{2 k-1}
\end{gathered}
$$




$$
\begin{gathered}
\sum_{n=1}^{N}\left(\mathscr{L} \boldsymbol{\eta}, \tau \mathbf{M}^{-1} \mathscr{L} \boldsymbol{\eta}\right)_{I_{n}} \leqslant c(\mathbf{u}) \Delta t^{2 k-1} \\
\mathscr{E}\left(\boldsymbol{\eta}\left(T^{-}\right)\right)+\mathscr{E}\left(\boldsymbol{\eta}\left(0^{+}\right)\right) \\
+\sum_{n=1}^{N-1}\left\{\mathscr{E}\left(\boldsymbol{\eta}\left(t_{n}^{-}\right)\right)+\mathscr{E}\left(\boldsymbol{\eta}\left(t_{n}^{+}\right)\right)\right\} \leqslant c(\mathbf{u}) \Delta t^{2 k-1}
\end{gathered}
$$

where $c(\mathbf{u})$ is independent of $\Delta t$. Then,

$$
\|\mathrm{e}\|^{2} \leqslant c(\mathbf{u}) \Delta t^{2 k-1}
$$

The following lemmas aid in the proof of Theorem 2 .

Lemma 3

$$
\begin{aligned}
& \sum_{n=1}^{N}\left(\dot{\mathbf{e}}^{h}, \mathbf{M} \ddot{\eta}\right)_{I_{n}}+\sum_{n=1}^{N-1} \dot{\mathbf{e}}^{h}\left(t_{n}^{+}\right) \cdot \mathbf{M} \llbracket \dot{\boldsymbol{\eta}}\left(t_{n}\right) \rrbracket+\dot{\mathbf{e}}^{h}\left(0^{+}\right) \cdot \mathbf{M} \dot{\boldsymbol{\eta}}\left(0^{+}\right) \\
& =-\sum_{n=1}^{N}\left(\ddot{\mathbf{e}}^{h}, \mathbf{M} \dot{\boldsymbol{\eta}}\right)_{I_{n}}-\sum_{n=1}^{N-1} \llbracket \dot{\mathbf{e}}^{h}\left(t_{n}\right) \rrbracket \cdot \mathbf{M} \dot{\eta}\left(t_{n}^{-}\right)+\dot{\mathbf{e}}^{h}\left(T^{-}\right) \cdot \mathbf{M} \dot{\boldsymbol{\eta}}\left(T^{-}\right)
\end{aligned}
$$

Proof

$$
\begin{aligned}
\left(\dot{\mathbf{e}}^{h}, \mathbf{M} \ddot{\mathbf{\eta}}\right)_{I_{n}}= & -\left(\ddot{\mathbf{e}}^{h}, \mathbf{M} \dot{\boldsymbol{\eta}}\right)_{I_{n}}+\int_{t_{n-1}}^{t_{n}} \frac{\mathrm{d}}{\mathrm{d} t}\left(\dot{\mathbf{e}}^{h} \cdot \mathbf{M} \dot{\boldsymbol{\eta}}\right) \mathrm{d} t \\
= & -\left(\ddot{\mathbf{e}}^{h}, \mathbf{M} \dot{\boldsymbol{\eta}}\right)_{I_{n}}+\dot{\mathbf{e}}^{h}\left(t_{n}^{-}\right) \cdot \mathbf{M} \dot{\eta}\left(t_{n}^{-}\right) \\
& +\dot{\mathbf{e}}^{h}\left(t_{n-1}^{+}\right) \cdot \mathbf{M} \dot{\eta}\left(t_{n-1}^{+}\right)
\end{aligned}
$$

So,

$$
\begin{aligned}
\left(\dot{\mathbf{e}}^{h}, \mathbf{M} \ddot{\eta}\right)_{I_{n}} & \left.+\dot{\mathbf{e}}^{h}\left(t_{n-1}^{+}\right) \cdot \mathbf{M} \llbracket \dot{\boldsymbol{\eta}}\left(t_{n-1}\right)\right] \\
& =-\left(\ddot{\mathbf{e}}^{h}, \mathbf{M} \dot{\boldsymbol{\eta}}\right)_{I_{n}}+\dot{\mathbf{e}}^{h}\left(t_{n}^{-}\right) \cdot \mathbf{M} \dot{\boldsymbol{\eta}}\left(t_{n}^{-}\right)-\dot{\mathbf{e}}^{h}\left(t_{n-1}^{+}\right) \cdot \mathbf{M} \dot{\boldsymbol{\eta}}\left(t_{n-1}^{-}\right)
\end{aligned}
$$

Thus,

$$
\begin{aligned}
\sum_{n=1}^{N} & \left.\left(\dot{\mathbf{e}}^{h}, \mathbf{M} \ddot{\mathbf{\eta}}\right)_{I_{n}}+\sum_{n=1}^{N-1} \dot{\mathbf{e}}^{h}\left(t_{n}^{+}\right) \cdot \mathbf{M} \llbracket \dot{\boldsymbol{\eta}}\left(t_{n}\right)\right]+\dot{\mathbf{e}}^{h}\left(0^{+}\right) \cdot \mathbf{M} \dot{\boldsymbol{\eta}}\left(0^{+}\right) \\
= & -\sum_{n=1}^{N}\left(\ddot{\mathbf{e}}^{h}, \mathbf{M} \dot{\boldsymbol{\eta}}\right)_{I_{n}}+\sum_{n=2}^{N}\left\{\dot{\mathbf{e}}^{h}\left(t_{n}^{-}\right) \cdot \mathbf{M} \dot{\boldsymbol{\eta}}\left(t_{n}^{-}\right)-\dot{\mathbf{e}}^{h}\left(t_{n-1}^{+}\right) \cdot \mathbf{M} \dot{\boldsymbol{\eta}}\left(t_{n-1}^{-}\right)\right\} \\
& +\dot{\mathbf{e}}^{h}\left(t_{1}^{-}\right) \cdot \mathbf{M} \dot{\boldsymbol{\eta}}\left(t_{1}^{-}\right) \\
= & -\sum_{n=1}^{N}\left(\ddot{\mathbf{e}}^{h}, \mathbf{M} \dot{\boldsymbol{\eta}}\right)_{I_{n}}-\sum_{n=1}^{N-1} \llbracket \dot{\mathbf{e}}^{h}\left(t_{n}\right) \rrbracket \cdot \mathbf{M} \dot{\boldsymbol{\eta}}\left(t_{n}^{-}\right)+\dot{\mathbf{e}}^{h}\left(T^{-}\right) \cdot \mathbf{M} \dot{\boldsymbol{\eta}}\left(T^{-}\right)
\end{aligned}
$$

Lemma 4

$$
\begin{aligned}
& \sum_{n=1}^{N}\left(\dot{\mathbf{e}}^{h}, \mathbf{K} \boldsymbol{\eta}\right)_{I_{n}}+\sum_{n=1}^{N-1} \mathbf{e}^{h}\left(t_{n}^{+}\right) \cdot \mathbf{K} \llbracket \boldsymbol{\eta}\left(t_{n}\right) \rrbracket+\mathbf{e}^{h}\left(0^{+}\right) \cdot \mathbf{K} \boldsymbol{\eta}\left(0^{+}\right) \\
= & -\sum_{n=1}^{N}\left(\mathbf{e}^{h}, \mathbf{K} \dot{\boldsymbol{\eta}}\right)_{I_{n}}-\sum_{n=1}^{N-1} \llbracket \mathbf{e}^{h}\left(t_{n}\right) \rrbracket \cdot \mathbf{K} \boldsymbol{\eta}\left(t_{n}^{-}\right)+\mathbf{e}^{h}\left(T^{-}\right) \cdot \mathbf{K} \boldsymbol{\eta}\left(T^{-}\right)
\end{aligned}
$$


Proof. The proof follows the same steps used to prove Lemma 3 and so is omitted.

Lemma 5

$$
\begin{gathered}
-\sum_{n=1}^{N-1}\left\{\llbracket \dot{\mathbf{e}}^{h}\left(t_{n}\right) \rrbracket \cdot \mathbf{M} \dot{\eta}\left(t_{n}^{-}\right)+\llbracket \mathbf{e}^{h}\left(t_{n}\right) \rrbracket \cdot \mathbf{K} \boldsymbol{\eta}\left(t_{n}^{-}\right)\right\}+\dot{\mathbf{e}}^{h}\left(T^{-}\right) \cdot \mathbf{M} \dot{\eta}\left(T^{-}\right)+\mathbf{e}^{h}\left(T^{-}\right) \cdot \mathbf{K} \boldsymbol{\eta}\left(T^{-}\right) \\
\leqslant \frac{1}{2}\left[\sum_{n=1}^{N-1}\left\{\mathscr{E}\left(\llbracket \mathbf{e}^{h}\left(t_{n}\right) \rrbracket\right)+4 \mathscr{E}\left(\boldsymbol{\eta}\left(t_{n}^{-}\right)\right)\right\}+\mathscr{E}\left(\mathbf{e}^{h}\left(T^{-}\right)\right)+4 \mathscr{E}\left(\boldsymbol{\eta}\left(T^{-}\right)\right)\right]
\end{gathered}
$$

Proof. Using the triangle inequality,

$$
\begin{aligned}
&-\sum_{n=1}^{N-1}\left\{\llbracket \dot{\mathbf{e}}^{h}\left(t_{n}\right) \rrbracket \cdot \mathbf{M} \dot{\eta}\left(t_{n}^{-}\right)+\llbracket \mathbf{e}^{h}\left(t_{n}\right) \rrbracket \cdot \mathbf{K} \boldsymbol{\eta}\left(t_{n}^{-}\right)\right\} \\
&+ \dot{\mathbf{e}}^{h}\left(T^{-}\right) \cdot \mathbf{M} \dot{\eta}\left(T^{-}\right)+\mathbf{e}^{h}\left(T^{-}\right) \cdot \mathbf{K} \boldsymbol{\eta}\left(T^{-}\right) \\
& \leqslant \frac{1}{2}\left[\sum _ { n = 1 } ^ { N - 1 } \left\{\frac{1}{2} \llbracket \dot{\mathbf{e}}^{h}\left(t_{n}\right) \rrbracket \cdot \mathbf{M} \llbracket \dot{\mathbf{e}}^{h}\left(t_{n}\right) \rrbracket+2 \dot{\boldsymbol{\eta}}\left(t_{n}^{-}\right) \cdot \mathbf{M} \dot{\eta}\left(t_{n}^{-}\right)\right.\right. \\
&\left.+\frac{1}{2} \llbracket \mathbf{e}^{h}\left(t_{n}\right) \rrbracket \cdot \mathbf{K} \llbracket \mathbf{e}^{h}\left(t_{n}\right) \rrbracket+2 \boldsymbol{\eta}\left(t_{n}^{-}\right) \cdot \mathbf{K} \boldsymbol{\eta}\left(t_{n}^{-}\right)\right\} \\
&+\frac{1}{2} \dot{\mathbf{e}}^{h}\left(T^{-}\right) \cdot \mathbf{M} \dot{\mathbf{e}}^{h}\left(T^{-}\right)+2 \dot{\boldsymbol{\eta}}\left(T^{-}\right) \cdot \mathbf{M} \dot{\boldsymbol{\eta}}\left(T^{-}\right) \\
&\left.+\frac{1}{2} \mathbf{e}^{h}\left(T^{-}\right) \cdot \mathbf{K} \mathbf{e}^{h}\left(T^{-}\right)+2 \eta\left(T^{-}\right) \cdot \mathbf{K} \boldsymbol{\eta}\left(T^{-}\right)\right] \\
&= \frac{1}{2}\left[\sum_{n=1}^{N-1}\left\{\mathscr{E}\left(\llbracket \mathbf{e}^{h}\left(t_{n}\right) \rrbracket\right)+4 \mathscr{E}\left(\boldsymbol{\eta}\left(t_{n}^{-}\right)\right)\right\}\right. \\
&\left.+\mathscr{E}\left(\mathbf{e}^{h}\left(T^{-}\right)\right)+4 \mathscr{E}\left(\boldsymbol{\eta}\left(T^{-}\right)\right)\right]
\end{aligned}
$$

The proof of Theorem 2 proceeds as follows:

$$
\begin{aligned}
\left\|\mathbf{e}^{h}\right\|= & b_{\mathrm{GLS}}\left(\mathbf{e}^{h}, \mathbf{e}^{h}\right) \quad(\text { by }(25)) \\
= & b_{\mathrm{GLS}}\left(\mathbf{e}^{h}, \mathbf{e}-\boldsymbol{\eta}\right) \quad(\text { by }(33)) \\
= & -b_{\mathrm{GLS}}\left(\mathbf{e}^{h}, \boldsymbol{\eta}\right) \quad(\text { by }(21)) \\
\leqslant & \left|b_{\mathrm{GLS}}\left(\mathbf{e}^{h}, \boldsymbol{\eta}\right)\right| \\
= & \mid \sum_{n=1}^{N}\left\{\left(\dot{\mathbf{e}}^{h}, \mathbf{M} \ddot{\boldsymbol{\eta}}\right)_{I_{n}}+\left(\dot{\mathbf{e}}^{h}, \mathbf{C} \dot{\boldsymbol{\eta}}\right)_{I_{n}}+\left(\dot{\mathbf{e}}^{h}, \mathbf{K} \boldsymbol{\eta}\right)_{I_{n}}\right. \\
& \left.+\left(\mathscr{L} \mathbf{e}^{h}, \tau \mathbf{M}^{-1} \mathscr{L} \boldsymbol{\eta}\right)_{I_{n}}\right\} \\
& \left.+\sum_{n=1}^{N-1}\left\{\dot{\mathbf{e}}^{h}\left(t_{n}^{+}\right) \cdot \mathbf{M} \llbracket \dot{\boldsymbol{\eta}}\left(t_{n}\right)\right]+\mathbf{e}^{h}\left(t_{n}^{+}\right) \cdot \mathbf{K}\left[\boldsymbol{\eta}\left(t_{n}\right)\right]\right\} \\
& +\dot{\mathbf{e}}^{h}\left(0^{+}\right) \cdot \mathbf{M} \dot{\eta}\left(0^{+}\right)+\mathbf{e}^{h}\left(0^{+}\right) \cdot \mathbf{K} \boldsymbol{\eta}\left(0^{+}\right) \mid \quad \text { (by (19)) }
\end{aligned}
$$




$$
\begin{aligned}
& =\mid \sum_{n=1}^{N}\left\{-\left(\mathscr{L} \mathbf{e}^{h}, \dot{\eta}\right)_{I_{n}}+\left(\mathscr{L} \mathbf{e}^{h}, \tau \mathbf{M}^{-1} \mathscr{L}_{\boldsymbol{\eta}}\right)_{I_{n}}+2\left(\dot{e}^{h}, \mathbf{C} \dot{\eta}\right)_{I_{n}}\right\} \\
& -\sum_{n=1}^{N-1}\left\{\llbracket \dot{e}^{h}\left(t_{n}\right) \rrbracket \cdot \mathbf{M} \dot{\eta}\left(t_{n}^{-}\right)+\llbracket \mathbf{e}^{h}\left(t_{n}\right) \rrbracket \cdot \mathbf{K} \boldsymbol{\eta}\left(t_{n}^{-}\right)\right\} \\
& +\dot{\mathbf{e}}^{h}\left(T^{-}\right) \cdot \mathbf{M} \dot{\eta}\left(T^{-}\right)+\mathbf{e}^{h}\left(T^{-}\right) \cdot \mathbf{K} \eta\left(T^{-}\right) \mid \quad \text { (by Lemmas } 3 \text { and 4) } \\
& \leqslant \sum_{n=1}^{N}\left\{\frac{1}{4}\left(\mathscr{L} \mathbf{e}^{h}, \tau \mathbf{M}^{-1} \mathscr{L} \mathbf{e}^{n}\right)_{I_{n}}+\left(\dot{\eta}, \tau^{-1} \mathbf{M} \dot{\eta}\right)_{I_{n}}\right. \\
& +\frac{1}{4}\left(\mathscr{L} \mathbf{e}^{h}, \tau \mathbf{M}^{-1} \mathscr{L} \mathbf{e}^{h}\right)_{I_{n}}+\left(\mathscr{L} \eta, \tau \mathbf{M}^{-1} \mathscr{L} \boldsymbol{\eta}\right)_{I_{n}} \\
& \left.+\frac{1}{2}\left(\dot{\mathrm{e}}^{h}, \mathbf{C} \dot{\mathrm{e}}^{h}\right)_{I_{n}}+2(\dot{\boldsymbol{\eta}}, \mathbf{C} \dot{\eta})_{I_{n}}\right\} \\
& +\frac{1}{2} \sum_{n=1}^{N-1} \mathscr{E}\left(\llbracket \mathrm{e}^{n}\left(t_{n}\right) \rrbracket\right)+2 \sum_{n=1}^{N-1} \mathscr{E}\left(\eta\left(t_{n}^{-}\right)\right) \\
& +\frac{1}{2} \mathscr{E}\left(\mathrm{e}^{h}\left(T^{-}\right)\right)+2 \mathscr{E}\left(\mathfrak{\eta}\left(T^{-}\right)\right) \quad \text { (by Lemma 5) }
\end{aligned}
$$

The terms involving $\mathrm{e}^{h}$ may be subsumed by the left-hand side. The interpolation estimates, (38)-(41), then yield

$$
\left\|\mid \mathbf{e}^{h}\right\|^{2} \leqslant c(\mathbf{u}) \Delta t^{2 k-1}
$$

Also, from the interpolation estimates and (24),

$$
\|\| \eta \|^{2} \leqslant c(\mathbf{u}) \Delta t^{2 k-1}
$$

By the triangle inequality,

$$
\left.\|\| \mathbf{e}\left\|\left.\right|^{2} \leqslant 2\right\|\left\|\mathbf{e}^{h}\right\|\right|^{2}+2\|\| \eta \|^{2} \leqslant c(\mathbf{u}) \Delta t^{2 k-1}
$$

which completes the proof.

It is important to emphasize that the least-squares operator is needed to bound the term, $\left(\mathscr{L} \mathbf{e}^{h}, \dot{\eta}\right)_{I_{n}}$. (See (50) for the first occurrence of this term.) If the finite element space is restricted to linear elements, then the $\mathrm{e}^{h}$ contributions produced by splitting $\left(\mathscr{L} \mathrm{e}^{h}, \dot{\eta}\right)_{I_{n}}$ can be subsumed by the left-hand side of $(50)$ without the use of the least-squares operator.

\section{A TWO-FIELD TIME FINITE ELEMENT FORMULATION}

\subsection{Variational equations}

With $\mathscr{P}^{k}$ defined as in the previous section, the finite element interpolation functions for the two-field formulation are:

trial displacement and displacement weighting functions

$$
\mathscr{S}_{1}^{h}=\left\{u_{1}^{h} \in \bigcup_{n=1}^{N}\left(\mathscr{P}^{k}\left(I_{n}\right)\right)^{n_{\bullet q}}\right\}
$$


trial velocity and velocity weighting functions

$$
\mathscr{S}_{2}^{h}=\left\{\mathbf{u}_{2}^{h} \in \bigcup_{n=1}^{N}\left(\mathscr{P}^{l}\left(I_{n}\right)\right)^{n_{\text {eq }}}\right\}
$$

The statement of the time-discontinuous Galerkin method for the two-field formulation is: Find $\mathbf{U}^{h}=\left\{\mathbf{u}_{1}^{h}, \mathbf{u}_{2}^{h}\right\} \in \mathscr{S}_{1}^{h} \times \mathscr{S}_{2}^{h}$ such that for all $\mathbf{W}^{h}=\left\{\mathbf{w}_{1}^{h}, \mathbf{w}_{2}^{h}\right\} \in \mathscr{S}_{1}^{h} \times \mathscr{S}_{2}^{h}$,

$$
B_{\mathrm{DG}}\left(\mathbf{W}^{h}, \mathbf{U}^{h}\right)_{n}=L_{\mathrm{DG}}\left(\mathbf{W}^{h}\right)_{n}, \quad n=1,2, \ldots, N
$$

where

$$
\begin{aligned}
B_{\mathrm{DG}}\left(\mathbf{W}^{h}, \mathbf{U}^{h}\right)_{n}= & \left(\mathbf{w}_{2}^{h}, \mathscr{L}_{1} \mathbf{U}^{h}\right)_{I_{n}}+\left(\mathbf{w}_{1}^{h}, \mathbf{K} \mathscr{L}_{2} \mathbf{U}^{h}\right)_{I_{n}} \\
& +\mathbf{w}_{2}^{h}\left(t_{n-1}^{+}\right) \cdot \mathbf{M} \mathbf{u}_{2}^{h}\left(t_{n-1}^{+}\right) \\
& +\mathbf{w}_{1}^{h}\left(t_{n-1}^{+}\right) \cdot \mathbf{K} \mathbf{u}_{1}^{h}\left(t_{n-1}^{+}\right) \\
L_{\mathrm{DG}}\left(\mathbf{W}^{h}\right)_{n}= & \left(\mathbf{w}_{2}^{h}, \mathbf{F}\right)_{I_{n}} \\
& +\mathbf{w}_{2}^{h}\left(t_{n-1}^{+}\right) \cdot \mathbf{M u}_{2}^{h}\left(t_{n-1}^{-}\right) \\
& +\mathbf{w}_{1}^{h}\left(t_{n-1}^{+}\right) \cdot \mathbf{K} \mathbf{u}_{1}^{h}\left(t_{n-1}^{-}\right), \quad n=2,3, \ldots, N \\
L_{\mathrm{DG}}\left(\mathbf{W}^{h}\right)_{1}= & \left(\mathbf{w}_{2}^{h}, \mathbf{F}\right)_{I_{1}} \\
& +\mathbf{w}_{2}^{h}\left(0^{+}\right) \cdot \mathbf{M} \mathbf{v}_{0}+\mathbf{w}_{1}^{h}\left(0^{+}\right) \cdot \mathbf{K} \mathbf{d}_{0}
\end{aligned}
$$

and

$$
\begin{aligned}
\mathscr{L}_{1} \mathbf{U}^{h} & =\mathbf{M} \dot{\mathbf{u}}_{2}^{h}+\mathbf{C} \mathbf{u}_{2}^{h}+\mathbf{K} \mathbf{u}_{1}^{h} \\
\mathscr{L}_{2} \mathbf{U}^{h} & =\dot{\mathbf{u}}_{1}^{h}-\mathbf{u}_{2}^{h}
\end{aligned}
$$

As with the single-field formulation, stability can be proved for the two-field version of the time-discontinuous Galerkin formulation, but convergence has been proved only for $k \leqslant 1$ and $l \leqslant 1$.

The statement of the time-discontinuous Galerkin/least-squares method for the two-field formulation is:

Find $\mathbf{U}^{h}=\left\{\mathbf{u}_{1}^{h}, \mathbf{u}_{2}^{h}\right\} \in \mathscr{S}_{1}^{h} \times \mathscr{S}_{2}^{h}$ such that for all $\mathbf{W}^{h}=\left\{\mathbf{w}_{1}^{h}, \mathbf{w}_{2}^{h}\right\} \in \mathscr{S}_{1}^{h} \times \mathscr{S}_{2}^{h}$

$$
B_{\mathrm{GLS}}\left(\mathbf{W}^{h}, \mathbf{U}^{h}\right)_{n}=L_{\mathrm{GLS}}\left(\mathbf{W}^{h}\right)_{n}, \quad n=1,2, \ldots, N
$$

where

$$
\begin{aligned}
B_{\mathrm{GLS}}\left(\mathbf{W}^{h}, \mathbf{U}^{h}\right)_{n}= & B_{\mathrm{DG}}\left(\mathbf{W}^{h}, \mathbf{U}^{h}\right)_{n} \\
& +\left(\mathscr{L}_{1} \mathbf{W}^{h}, \tau_{1} \mathbf{M}^{-1} \mathscr{L}_{1} \mathbf{U}^{h}\right)_{I_{n}} \\
& +\left(\mathscr{L}_{2} \mathbf{W}^{h}, \tau_{2} \mathbf{K} \mathscr{L}_{2} \mathbf{U}^{h}\right)_{I_{n}} \\
L_{\mathrm{GLS}}\left(\mathbf{W}^{h}\right)_{n}= & L_{\mathrm{DG}}\left(\mathbf{W}^{h}\right)_{n}+\left(\mathscr{L}_{1} \mathbf{W}^{h}, \tau_{1} \mathbf{M}^{-1} \mathbf{F}\right)_{I_{n}}
\end{aligned}
$$

and $\tau_{1}$ and $\tau_{2}$ are intrinsic time-scale parameters.

\subsection{Stability and convergence analyses}

The stability and convergence proofs for the two-field formulation are similar to those for the single-field formulation. Hence, the steps presented in the two-field formulation proofs are those which differ from the steps taken in Section 2.2. 
A sufficiently smooth exact solution of (1)-(3), $\mathbf{U}=\{\mathbf{u}, \dot{\mathbf{u}}\}$, satisfies

$$
B_{\mathrm{GLS}}\left(\mathbf{W}^{h}, \mathbf{U}\right)_{n}=L_{\mathrm{GLS}}\left(\mathbf{W}^{h}\right)_{n}
$$

for all $\mathbf{W}^{h} \in \mathscr{S}_{1}^{h} \times \mathscr{S}_{2}^{h}$ and $n=1,2, \ldots, N$. This may be seen from the Euler-Lagrange form of (62):

$$
\begin{aligned}
0= & B_{\mathrm{GLS}}\left(\mathbf{W}^{h}, \mathbf{U}^{h}\right)_{n}-L_{\mathrm{GLS}}\left(\mathbf{W}^{h}\right)_{n} \\
= & \left(\mathbf{w}_{2}^{h}+\tau_{1} \mathbf{M}^{-1} \mathscr{L}_{1} \mathbf{W}^{h}, \mathscr{L}_{1} \mathbf{U}^{h}-\mathbf{F}\right)_{I_{n}} \\
& +\left(\mathbf{w}_{1}^{h}+\tau_{2} \mathscr{L}_{2} \mathbf{W}^{h}, \mathbf{K} \mathscr{L}_{2} \mathbf{U}^{h}\right)_{I_{n}} \\
& +\mathbf{w}_{2}^{h}\left(t_{n-1}^{+}\right) \cdot \mathbf{M} \llbracket \mathbf{u}_{2}^{h}\left(t_{n-1}\right) \rrbracket \\
& +\mathbf{w}_{1}^{h}\left(t_{n-1}^{+}\right) \cdot \mathbf{K} \llbracket \mathbf{u}_{1}^{h}\left(t_{n-1}\right) \rrbracket
\end{aligned}
$$

Summing (62) over the time intervals and rearranging terms yields:

$$
B_{\mathrm{GLS}}\left(\mathbf{W}^{h}, \mathbf{U}^{h}\right)=L_{\mathrm{GLS}}\left(\mathbf{W}^{h}\right)
$$

where

$$
\begin{aligned}
B_{\mathrm{GLS}}\left(\mathbf{W}^{h}, \mathbf{U}^{h}\right)= & \sum_{n=1}^{N}\left\{\left(\mathbf{w}_{2}^{h}, \mathscr{L}_{1} \mathbf{U}^{h}\right)_{I_{n}}+\left(\mathbf{w}_{1}^{h}, \mathbf{K} \mathscr{L}_{2} \mathbf{U}^{h}\right)_{I_{n}}\right. \\
& +\left(\mathscr{L}_{1} \mathbf{W}^{h}, \tau_{1} \mathbf{M}^{-1} \mathscr{L}_{1} \mathbf{U}^{h}\right)_{I_{n}} \\
& \left.+\left(\mathscr{L}_{2} \mathbf{W}^{h}, \tau_{2} \mathbf{K} \mathscr{L}_{2} \mathbf{U}^{h}\right)_{I_{n}}\right\} \\
& +\sum_{n=1}^{N-1}\left\{\mathbf{w}_{2}^{h}\left(t_{n}^{+}\right) \cdot \mathbf{M} \llbracket \mathbf{u}_{2}^{h}\left(t_{n}\right)\right] \\
& \left.\left.+\mathbf{w}_{1}^{h}\left(t_{n}^{+}\right) \cdot \mathbf{K} \llbracket \mathbf{u}_{1}^{h}\left(t_{n}\right)\right]\right\} \\
& +\mathbf{w}_{2}^{h}\left(0^{+}\right) \cdot \mathbf{M} \mathbf{u}_{2}^{h}\left(0^{+}\right) \\
& +\mathbf{w}_{1}^{h}\left(0^{+}\right) \cdot \mathbf{K} \mathbf{u}_{1}^{h}\left(0^{+}\right) \\
& \sum_{n=1}^{N}\left\{\left(\mathbf{w}_{2}^{h}, \mathbf{F}\right)_{I_{n}}+\left(\mathscr{L}^{h}, \tau_{1} \mathbf{M}^{-1} \mathbf{F}\right)_{I_{n}}\right\} \\
& +\mathbf{w}_{2}^{h}\left(0^{+}\right) \cdot \mathbf{M} \mathbf{v}_{0}+\mathbf{w}_{1}^{h}\left(0^{+}\right) \cdot \mathbf{K} \mathbf{d}_{0}
\end{aligned}
$$

By (65), (67) is also satisfied by substituting a sufficiently smooth exact solution, $\mathbf{U}$, for $\mathbf{U}^{\text {h }}$. Thus, for all $\mathbf{W}^{h} \in \mathscr{S}_{1}^{h} \times \mathscr{S}_{2}^{h}$,

$$
B_{\mathrm{GLS}}\left(\mathbf{W}^{h}, \mathbf{E}\right)=0
$$

where

$$
\mathbf{E}=\mathbf{U}^{h}-\mathbf{U}
$$

This is the consistency condition for the two-field formulation.

3.2.1. Stability. The total energy for the two-field formulation is given as:

$$
\mathscr{E}\left(\mathbf{W}^{h}\right)=\frac{1}{2} \mathbf{w}_{2}^{h} \cdot \mathbf{M} \mathbf{w}_{2}^{h}+\frac{1}{2} \mathbf{w}_{1}^{h} \cdot \mathbf{K} \mathbf{w}_{1}^{h}
$$


The norm associated with (67) is defined by:

$$
\begin{aligned}
\left\|\mathbf{W}^{h}\right\|= & \mathscr{E}\left(\mathbf{W}^{h}\left(T^{-}\right)\right)+\mathscr{E}\left(\mathbf{W}^{h}\left(0^{+}\right)\right)+\sum_{n=1}^{N-1} \mathscr{E}\left(\left[\mathbf{W}^{h}\left(t_{n}\right)\right]\right) \\
& +\sum_{n=1}^{N}\left\{\left(\mathbf{w}_{2}^{h}, \mathbf{C} \mathbf{W}_{2}^{h}\right)_{I_{n}}+\left(\mathscr{L}_{1} \mathbf{W}^{h}, \tau_{1} \mathbf{M}^{-1} \mathscr{L}_{1} \mathbf{W}^{h}\right)_{I_{n}}\right. \\
& \left.+\left(\mathscr{L}_{2} \mathbf{W}^{h}, \tau_{2} \mathscr{L}_{2} \mathbf{W}^{h}\right)_{I_{n}}\right\}
\end{aligned}
$$

Lemma 6 (Stability condition)

$$
B_{\mathrm{GLS}}\left(\mathbf{W}^{h} ; \mathbf{W}^{h}\right)=\left\|\mid \mathbf{W}^{h}\right\|^{2}
$$

Proof. The proof of the stability condition for the two-field formulation is similar to that for Lemma 1 and so is omitted.

Theorem 3 (Energy decay inequality). In the absence of external forces, i.e. $\mathbf{F}=\mathbf{0}$, the total energy at the end of a time interval, e.g. $\mathscr{E}\left(\mathbf{U}^{h}\left(t_{n}^{-}\right)\right)$, computed using the Galerkin/least-squares method, (62), is bounded by the initial total energy. That is,

$$
\mathscr{E}\left(\mathbf{U}^{h}\left(t_{n}^{-}\right)\right) \leqslant \mathscr{E}\left(\mathbf{U}_{0}\right), \quad n=1,2, \ldots, N
$$

where $\mathscr{E}\left(\mathbf{U}_{0}\right)$ is the initial total energy.

Proof. The same steps to prove stability for the single-field formulation are taken to prove stability for the two-field case and thus are omitted.

3.2.2. Convergence. Let $\Pi \mathbf{U}=\{\Pi u, \Pi \dot{\mathbf{u}}\} \in \mathscr{S}_{1}^{h} \times \mathscr{S}_{2}^{h}$ denote an interpolant of $\mathbf{U}$. Then,

$$
\mathbf{E}=\mathbf{E}^{h}+\mathbf{H}
$$

where

$$
\begin{aligned}
\mathbf{E}^{h} & =\left(\mathbf{U}^{h}-\Pi \mathbf{U}\right) \in \mathscr{P}_{1}^{h} \times \mathscr{S}_{2}^{h} \\
\mathbf{H} & =\boldsymbol{\Pi} \mathbf{U}-\mathbf{U}
\end{aligned}
$$

In components,

$$
\mathbf{E}=\left\{\mathbf{e}_{1}, \mathbf{e}_{2}\right\}, \quad \mathbf{E}^{h}=\left\{\mathbf{e}_{1}^{h}, \mathbf{e}_{2}^{h}\right\} \quad \text { and } \quad \mathbf{H}=\left\{\boldsymbol{\eta}_{1}, \boldsymbol{\eta}_{2}\right\}
$$

Theorem 4 (Error estimates). Assume $\tau_{1}$ and $\tau_{2}$ satisfy:

$$
\begin{aligned}
& c_{1} \Delta t \leqslant \tau_{1} \leqslant c_{2} \Delta t \\
& c_{1} \Delta t \leqslant \tau_{2} \leqslant c_{2} \Delta t
\end{aligned}
$$

where $c_{1}$ and $c_{2}$ are positive constants $\left(c_{2}>c_{1}\right)$.

Assuming $\mathbf{U} \in\left(H^{k+1}(I)\right)^{n_{e q}} \times\left(H^{l+1}(I)\right)^{n_{e q}}$, then the interpolation error, $\mathbf{H}$, satisfies:

$$
\begin{aligned}
\sum_{n=1}^{N}\left(\boldsymbol{\eta}_{2}, \mathbf{C} \eta_{2}\right)_{I_{n}} & \leqslant c(\mathbf{U}) \Delta t^{2 l+2} \\
\sum_{n=1}^{N}\left(\boldsymbol{\eta}_{2}, \tau_{1}^{-1} \mathbf{M} \boldsymbol{\eta}_{2}\right)_{I_{n}} & \leqslant c(\mathbf{U}) \Delta t^{2 l+1}
\end{aligned}
$$




$$
\begin{gathered}
\sum_{n=1}^{N}\left(\boldsymbol{\eta}_{1}, \tau_{2}^{-1} \mathbf{K} \boldsymbol{\eta}_{1}\right)_{I_{n}} \leqslant c(\mathbf{U}) \Delta t^{2 k+1} \\
\sum_{n=1}^{N}\left(\mathscr{L}_{1} \mathbf{H}, \tau_{1} \mathbf{M}^{-1} \mathscr{L}_{1} \mathbf{H}\right)_{I_{n}} \leqslant c(\mathbf{U}) \Delta t^{\min (2 k+3,2 l+1)} \\
\sum_{n=1}^{N}\left(\mathscr{L}_{2} \mathbf{H}, \tau_{2} \mathbf{K} \mathscr{L}_{2} \mathbf{H}\right)_{I_{n}} \leqslant c(\mathbf{U}) \Delta t^{\min (2 k+1,2 l+3)} \\
\mathscr{E}\left(\boldsymbol{\eta}\left(T^{-}\right)+\mathscr{E}\left(\boldsymbol{\eta}\left(0^{+}\right)\right)\right. \\
+\sum_{n=1}^{N-1}\left\{\mathscr{E}\left(\boldsymbol{\eta}\left(t_{n}^{-}\right)\right)+\mathscr{E}\left(\boldsymbol{\eta}\left(t_{n}^{+}\right)\right)\right\} \leqslant c(\mathbf{U}) \Delta t^{\min (2 k+1,2 l+1)}
\end{gathered}
$$

where $c(\mathbf{U})$ is independent of $\Delta t$. Then,

$$
\|\mathbf{E}\|^{2} \leqslant c(\mathbf{U}) \Delta t^{\min (2 k+1,2 l+1)}
$$

The next three lemmas aid in the proof of Theorem 4 . They are given without proof since the steps needed to prove them are identical to those in the proofs of Lemmas 3-5.

Lemma 7

$$
\begin{aligned}
& \sum_{n=1}^{N}\left(\mathbf{e}_{2}^{h}, \mathbf{M} \dot{\eta}_{2}\right)_{I_{n}}+\sum_{n=1}^{N-1} \mathbf{e}_{2}^{h}\left(t_{n}^{+}\right) \cdot \mathbf{M} \llbracket \boldsymbol{\eta}_{2}\left(t_{n}\right) \rrbracket+\mathbf{e}_{2}^{h}\left(0^{+}\right) \cdot \mathbf{M} \boldsymbol{\eta}_{2}\left(0^{+}\right) \\
& =-\sum_{n=1}^{N}\left(\dot{\mathbf{e}}_{2}^{h}, \mathbf{M} \boldsymbol{\eta}_{2}\right)_{I_{n}}-\sum_{n=1}^{N-1} \llbracket \mathbf{e}_{2}^{h}\left(t_{n}\right) \rrbracket \cdot \mathbf{M} \boldsymbol{\eta}_{2}\left(t_{n}^{-}\right)+\mathbf{e}_{2}^{h}\left(T^{-}\right) \cdot \mathbf{M} \boldsymbol{\eta}_{2}\left(T^{-}\right)
\end{aligned}
$$

Lemma 8

$$
\begin{aligned}
& \sum_{n=1}^{N}\left(\mathbf{e}_{1}^{h}, \mathbf{K} \dot{\boldsymbol{\eta}}_{1}\right)_{I_{n}}+\sum_{n=1}^{N-1} \mathbf{e}_{1}^{h}\left(t_{n}^{+}\right) \cdot \mathbf{K} \llbracket \boldsymbol{\eta}_{1}\left(t_{n}\right) \rrbracket+\mathbf{e}_{1}^{h}\left(0^{+}\right) \cdot \mathbf{K} \boldsymbol{\eta}_{1}\left(0^{+}\right) \\
& =-\sum_{n=1}^{N}\left(\dot{\mathbf{e}}_{1}^{h}, \mathbf{K} \boldsymbol{\eta}_{1}\right)_{I_{n}}-\sum_{n=1}^{N-1} \llbracket \mathbf{e}_{1}^{h}\left(t_{n}\right) \rrbracket \cdot \mathbf{K} \boldsymbol{\eta}_{1}\left(t_{n}^{-}\right)+\mathbf{e}_{1}^{h}\left(T^{-}\right) \cdot \mathbf{K} \boldsymbol{\eta}_{1}\left(T^{-}\right)
\end{aligned}
$$

Lemma 9

$$
\begin{aligned}
\left.-\sum_{n=1}^{N-1}\left\{\llbracket \mathbf{e}_{2}^{h}\left(t_{n}\right) \rrbracket \cdot \mathbf{M} \boldsymbol{\eta}_{2}\left(t_{n}^{-}\right)+\llbracket \mathbf{e}_{1}^{h}\left(t_{n}\right)\right] \cdot \mathbf{K} \boldsymbol{\eta}_{1}\left(t_{n}^{-}\right)\right\} \\
+\mathbf{e}_{2}^{h}\left(T^{-}\right) \cdot \mathbf{M} \boldsymbol{\eta}_{2}\left(T^{-}\right)+\mathbf{e}_{1}^{h}\left(T^{-}\right) \cdot \mathbf{K} \boldsymbol{\eta}_{1}\left(T^{-}\right) \\
\leqslant \frac{1}{2}\left[\sum_{n=1}^{N-1}\left\{\mathscr{E}\left(\llbracket \mathbf{E}^{h}\left(t_{n}\right) \rrbracket\right)+4 \mathscr{E}\left(\mathbf{H}\left(t_{n}^{-}\right)\right)\right\}\right. \\
\left.+\mathscr{E}\left(\mathbf{E}^{h}\left(T^{-}\right)\right)+4 \mathscr{E}\left(\mathbf{H}\left(T^{-}\right)\right)\right]
\end{aligned}
$$

The proof of Theorem 4 is as follows:

$$
\begin{aligned}
\left.\left\|\mathbf{E}^{h}\right\|\right|^{2} & =B_{\mathrm{GLS}}\left(\mathbf{E}^{h}, \mathbf{E}^{h}\right) & & (\text { by (74)) } \\
& =B_{\mathrm{GLS}}\left(\mathbf{E}^{h}, \mathbf{E}-\mathbf{H}\right) & & (\text { by (76)) } \\
& =-B_{\mathrm{GLS}}\left(\mathbf{E}^{h}, \mathbf{H}\right) & & (\text { by (70)) }
\end{aligned}
$$




$$
\begin{aligned}
& \leqslant\left|B_{\mathrm{GLS}}\left(\mathbf{E}^{h}, \mathbf{H}\right)\right| \\
& =\mid \sum_{n=1}^{N}\left\{\left(\mathbf{e}_{2}^{h}, \mathscr{L}_{1} \mathbf{H}\right)_{I_{n}}+\left(\mathbf{e}_{1}^{h}, \mathbf{K} \mathscr{L}_{2} \mathbf{H}\right)_{I_{n}}\right. \\
& +\left(\mathscr{L}_{1} \mathbf{E}^{h}, \tau_{1} \mathbf{M}^{-1} \mathscr{L}_{1} \mathbf{H}\right)_{I_{n}} \\
& \left.+\left(\mathscr{L}_{2} \mathbf{E}^{h}, \tau_{2} \mathbf{K} \mathscr{L}_{2} \mathbf{H}\right)_{I_{n}}\right\} \\
& +\sum_{n=1}^{N-1}\left\{\mathbf{e}_{2}^{h}\left(t_{n}^{+}\right) \cdot \mathbf{M} \llbracket \boldsymbol{\eta}_{2}\left(t_{n}\right) \rrbracket+\mathbf{e}_{1}^{h}\left(t_{n}^{+}\right) \cdot \mathbf{K} \llbracket \boldsymbol{\eta}_{1}\left(t_{n}\right) \rrbracket\right\} \\
& +\mathbf{e}_{2}^{h}\left(0^{+}\right) \cdot \mathbf{M} \eta_{2}\left(0^{+}\right)+\mathbf{e}_{1}^{h}\left(0^{+}\right) \cdot \mathbf{K} \eta_{1}\left(0^{+}\right) \mid \quad(\text { by }(68)) \\
& =\mid \sum_{n=1}^{N}\left\{-\left(\mathscr{L}_{\mathbf{1}} \mathbf{E}^{h}, \boldsymbol{\eta}_{2}\right)_{I_{n}}-\left(\mathscr{L}_{2} \mathbf{E}^{h}, \mathbf{K} \boldsymbol{\eta}_{\mathbf{1}}\right)_{I_{n}}\right. \\
& +\left(\mathscr{L}_{1} \mathbf{E}^{h}, \tau_{1} \mathbf{M}^{-1} \mathscr{L}_{1} \mathbf{H}\right)_{I_{n}}+\left(\mathscr{L}_{2} \mathbf{E}^{h}, \tau_{2} \mathbf{K} \mathscr{L}_{2} \mathbf{H}\right)_{I_{n}} \\
& \left.+2\left(\mathbf{e}_{2}^{h}, \mathbf{C \eta}_{2}\right)_{I_{n}}\right\} \\
& -\sum_{n=1}^{N-1}\left\{\llbracket \mathbf{e}_{2}^{h}\left(t_{n}\right) \rrbracket \cdot \mathbf{M} \boldsymbol{\eta}_{2}\left(t_{n}^{-}\right)+\llbracket\left[\mathbf{e}_{1}^{h}\left(t_{n}\right) \rrbracket \cdot \mathbf{K} \boldsymbol{\eta}_{1}\left(t_{n}^{-}\right)\right\}\right. \\
& +\mathbf{e}_{2}^{h}\left(T^{-}\right) \cdot \mathbf{M} \eta_{2}\left(T^{-}\right)+\mathbf{e}_{1}^{h}\left(T^{-}\right) \cdot \mathbf{K} \eta_{1}\left(T^{-}\right) \mid \quad \text { (by Lemmas } 7 \text { and } 8 \text { ) } \\
& \leqslant \sum_{n=1}^{N}\left\{\frac{1}{4}\left(\mathscr{L}_{1} \mathbf{E}^{h}, \tau_{1} \mathbf{M}^{-1} \mathscr{L}_{1} \mathbf{E}^{h}\right)_{I_{n}}+\left(\boldsymbol{\eta}_{2}, \tau_{1}^{-1} \mathbf{M} \boldsymbol{\eta}_{2}\right)_{I_{n}}\right. \\
& +\frac{1}{4}\left(\mathscr{L}_{2} \mathbf{E}^{h}, \tau_{2} \mathbf{K} \mathscr{L}_{2} \mathbf{E}^{h}\right)_{I_{n}}+\left(\boldsymbol{\eta}_{1}, \tau_{2}^{-1} \mathbf{K} \boldsymbol{\eta}_{1}\right)_{I_{n}} \\
& +\frac{1}{4}\left(\mathscr{L}_{1} \mathbf{E}^{h}, \tau_{1} \mathbf{M}^{-1} \mathscr{L}_{1} \mathbf{E}^{h}\right)_{I_{n}}+\left(\mathscr{L}_{1} \mathbf{H}, \tau_{1} \mathbf{M}^{-1} \mathscr{L}_{1} \mathbf{H}\right)_{I_{n}} \\
& +\frac{1}{4}\left(\mathscr{L}_{2} \mathbf{E}^{h}, \tau_{2} \mathbf{K} \mathscr{L}_{2} \mathbf{E}^{h}\right)_{I_{n}}+\left(\mathscr{L}_{2} \mathbf{H}, \tau_{2} \mathbf{K} \mathscr{L}_{2} \mathbf{H}\right)_{I_{n}} \\
& \left.+\frac{1}{2}\left(\mathbf{e}_{2}^{h}, \mathbf{C} \mathbf{e}_{2}^{h}\right)_{I_{n}}+2\left(\boldsymbol{\eta}_{2}, \mathbf{C} \boldsymbol{\eta}_{2}\right)_{I_{n}}\right\} \\
& +\frac{1}{2} \sum_{n=1}^{N-1} \mathscr{E}\left(\llbracket \mathbf{E}^{h}\left(t_{n}\right) \rrbracket\right)+2 \sum_{n=1}^{N-1} \mathscr{E}\left(\mathbf{H}\left(t_{n}^{-}\right)\right) \\
& +\frac{1}{2} \mathscr{E}\left(\mathbf{E}^{h}\left(T^{-}\right)\right)+2 \mathscr{E}\left(\mathbf{H}\left(T^{-}\right)\right) \quad \text { (by Lemma 9) }
\end{aligned}
$$

The terms involving $\mathbf{E}^{h}$ may be subsumed by the left-hand side. The interpolation estimates, (82)-(87), then yield

$$
\left.\left\|\mathbf{E}^{h}\right\|\right|^{2} \leqslant c(\mathrm{U}) \Delta t^{\min (2 k+1,2 l+1)}
$$

Also, from the interpolation estimates and (73),

$$
\|\mathbf{H}\| \|^{2} \leqslant c(\mathbf{U}) \Delta t^{\min (2 k+1,2 l+1)}
$$


By the triangle inequality:

$$
\left.\|\mathbf{E}\|\right|^{2} \leqslant 2\left\|\mathbf{E}^{h}\right\|^{2}+2\|\| \mathbf{H} \|\left.\right|^{2} \leqslant c(\mathbf{U}) \Delta t^{\min (2 k+1,2 l+1)}
$$

which compietes the proof.

The least-squares operators are needed to bound $\left|\left(\mathscr{L}_{1} \mathbf{E}^{\boldsymbol{h}}, \boldsymbol{\eta}_{2}\right)_{I_{n}}\right|$ and $\left|\left(\mathscr{L}_{2} \mathbf{E}^{h}, \mathbf{K} \boldsymbol{\eta}_{1}\right)_{\boldsymbol{I}_{n}}\right|$ in (92). Without the least-squares operators, convergence has been proved only for linear displacement and velocity interpolations.

\section{COMPARISON OF SINGLE-FIELD AND TWO-FIELD FORMULATIONS}

As measured in the $|\| \cdot|||$-norms, the convergence rates proved for the single-field (two-field) formulation are sharp (in the sense that the interpolation error contributions and the error contributions emanating from $\mathrm{e}^{h}\left(\mathbf{E}^{h}\right)$ have the same rates of convergence). If the velocity field interpolation is chosen to be one order less than that of the displacement field (i.e. $l=k-1$ ), then the two-field formulation reduces to that of the single-field. However, it is computationally convenient to choose equal-order interpolations for the velocity and displacement fields.

The analyses indicate that the intrinsic time-scale parameters, $\tau, \tau_{1}$ and $\tau_{2}$, are proportional to $\Delta t$, but these analyses are not sufficient to define the proportionality constants. Practical definitions of the time-scale parameters are given by

$$
\tau=\tau_{1}=\tau_{2}=\frac{1}{2} \Delta t
$$

If variable time steps are used, then $\Delta t$ is to be interpreted as a local value. That is, in (96), $\Delta t$ should be replaced by $\Delta t_{n}$.

\section{FINITE DIFFERENCE ANALYSES}

Stability properties and error estimates derived in Sections 2 and 3 are based on functional analysis techniques typical of most finite element analyses. In contrast, for semidiscrete formulations, classical finite difference techniques usually are used to derive stability and error measures. In this section, we present results from finite difference analyses of the time finite element methods. Particular emphasis is placed on the temporal accuracy and dissipative properties of the proposed methods. These results are compared with finite difference analysis results of several commonly used semidiscrete methods. For the single-field formulation, linear $(P 1)$ and quadratic $(P 2)$ interpolations are analysed. Four combinations of interpolations are studied within the twofield formulation: constant displacement and velocity $(P 0-P 0)$, linear displacement and constant velocity $(P 1-P 0)$, linear displacement and velocity $(P 1-P 1)$, and quadratic displacement and linear velocity $(P 2-P 1)$. Both the time-discontinuous Galerkin and the Galerkin/least-squares methods are investigated.

\subsection{Model problem and analytical measures}

To study the finite element formulations from a finite difference perspective, we start with the time finite element method for ordinary differential equations to assess the temporal behaviour of the algorithms. It suffices to consider the undamped, single-degree-of-freedom model problem:

$$
\begin{aligned}
\ddot{d}+\left(\omega^{h}\right)^{2} d & =0 \\
d(0) & =d_{0} \\
\dot{d}(0) & =v_{0}
\end{aligned}
$$


See Chapters 8 and 9 of Hughes $^{40}$ for an explication of the steps taken to obtain (97)-(99) from (1)-(3).

For purposes of analysis, it is convenient to express the values at the end of a time interval, e.g. $d^{h}\left(t_{n}^{-}\right)$and $v^{h}\left(t_{n}^{-}\right)$, in terms of the values at the end of the previous time interval. This can be written in the form:

$$
\left\{\begin{array}{c}
d^{h}\left(t_{n}^{-}\right) \\
\Delta t v^{h}\left(t_{n}^{-}\right)
\end{array}\right\}=\mathbf{A}\left\{\begin{array}{c}
d^{h}\left(t_{n-1}^{-}\right) \\
\Delta t v^{h}\left(t_{n-1}^{-}\right)
\end{array}\right\}
$$

where $\mathbf{A}$ is the amplification matrix of dimension $2 \times 2$. The analytical measures of interest are the spectral radius, local truncation error and numerical dissipation and dispersion. The spectral radius, $\rho(\mathbf{A})$, is defined by

$$
\rho(\mathbf{A})=\max \left|\lambda_{1}(\mathbf{A}), \lambda_{2}(\mathbf{A})\right|
$$

where $\lambda_{i}(\mathbf{A})$ denotes the $i$ th eigenvalue of $\mathbf{A}$. Calculating the displacement-difference stencil from $(100)$ results in

$$
d^{h}\left(t_{n+1}^{-}\right)-2 A_{1} d^{h}\left(t_{n}^{-}\right)+A_{2} d^{h}\left(t_{n-1}^{-}\right)=0
$$

where

$$
\begin{aligned}
& A_{1}=\frac{1}{2} \text { trace } \mathbf{A} \\
& A_{2}=\operatorname{det} \mathbf{A}
\end{aligned}
$$

The local truncation error, $\mathscr{T}(t)$, is defined by

$$
\mathscr{T}\left(t_{n}^{-}\right)=\Delta t^{-2}\left(d\left(t_{n}+\Delta t\right)-2 A_{1} d\left(t_{n}\right)+A_{2} d\left(t_{n}-\Delta t\right)\right)
$$

where $d$ is the exact solution to (97)-(99). Provided that the eigenvalues of $\mathbf{A}$ remain complex $\left(\lambda_{1,2}(A)=A \pm \mathrm{i} B, B \neq 0\right)$, the solution of (102) can be written as:

$$
d^{h}\left(t_{n}^{-}\right)=\exp \left(-\bar{\xi}^{h} \bar{\omega}^{h} t_{n}\right)\left(c_{1} \cos \left(\bar{\omega}^{h} t_{n}\right)+c_{2} \sin \left(\bar{\omega}^{h} t_{n}\right)\right)
$$

in which

$$
\begin{aligned}
\bar{\omega}^{h} & =\Delta t^{-1} \tan ^{-1}\left(\frac{B}{A}\right) \\
\bar{\xi}^{h} & =-\frac{\ln \rho}{\Delta t \bar{\omega}^{h}}
\end{aligned}
$$

where $\bar{\xi}^{h}$ is the algorithmic damping ratio, $\bar{\omega}^{h}$ is the approximate frequency and the coefficients $c_{1}$ and $c_{2}$ are determined from the initial conditions. The algorithmic damping ratio provides a measure of the numerical dissipation while the relative frequency error, calculated using

$$
\frac{\omega^{h}}{\bar{\omega}^{h}}-1
$$

is a measure of numerical dispersion. The relative frequency error may also be expressed as:

$$
\frac{\Omega}{\bar{\Omega}}-1
$$

where $\Omega=\Delta t \omega^{h}$ and $\bar{\Omega}=\Delta t \bar{\omega}^{h}$. For details on the derivation of the above analytical measures see Hughes $^{40}$ and Hilber. ${ }^{41}$ 


\subsection{Local truncation errors}

In a local truncation error expression, the power of the lowest-order term with respect to $\Delta t$ defines the convergence rate of the associated algorithm. The coefficient of this lowest-order term provides a measure of relative error magnitudes for comparing different algorithms.

Table I lists the lowest-order terms in the local truncation errors of the time-discontinuous Galerkin method using the six interpolation combinations described above. As is typical of time finite element methods, the convergence rates are odd powers of $\Delta t$. The $P 0-P 0, P 1-P 0$ and $P 1$ elements exhibit first-order accuracy, while third-order accuracy is achieved by the $P 1-P 1$, $P 2-P 1$ and $P 2$ elements. The displacement-difference stencils are identical for the time-discontinuous Galerkin formulation using $P 1-P 0$ and $P 1$ elements; thus, their local truncation error expressions are also identical. Similarly, the local truncation errors of $P 2-P 1$ and $P 2$ are identical. These equivalences are not surprising since, in the two-field formulation, the compatibility equation (61) is equivalent to calculating velocity from the displacement field in the singlefield formulation, provided $l=k-1$. The $P 0-P 0$ element, while convergent, is equivalent to the backward Euler method, which is a poor algorithm for accurately computing transient response. Also, owing to their low rates of convergence, the $P 1-P 0$ and $P 1$ elements are inappropriate methods for solving structural dynamics problems. In contrast, the three methods that exhibit third-order accuracy are useful transient solvers. We note that commonly used structural dynamics algorithms are second-order accurate.

Table II lists the lowest-order terms in the local truncation errors of the Galerkin/least-squares method using the same six interpolation combinations. Compared to these error measures, the error estimates obtained from the convergence analyses of Sections 2 and 3 are pessimistic for the model problem. Comparing the results in Tables II and I, note that the addition of the leastsquares terms does not degrade the convergence rate but does increase the error coefficient. If $\tau_{1}=\tau_{2}$, then the error coefficient for $P 1-P 1$ is twice that of $P 2-P 1$. In addition, if $\tau_{1}=\tau$, then the error coefficients of $P 2-P 1$ and $P 2$ are identical, as are the error coefficients for $P 1-P 0$ and $P 1$.

Table I. Local truncation errors for the time-discontinuous Galerkin method

\begin{tabular}{llcc}
\hline \multicolumn{1}{c}{ Interpolation } & Error rate & Error coefficient \\
\hline $\begin{array}{c}\text { Constant displacement, } \\
\text { Constant velocity }\end{array}$ & $(P 0-P 0)$ & $\Delta t$ & 1 \\
\hline $\begin{array}{c}\text { Linear displacement, } \\
\text { Constant velocity }\end{array}$ & $(P 1-P 0)$ & $\Delta t$ & $1 / 2$ \\
\hline $\begin{array}{c}\text { Linear displacement, } \\
\text { Linear velocity }\end{array}$ & $(P 1-P 1)$ & $\Delta t^{3}$ & $1 / 36$ \\
\hline $\begin{array}{l}\text { Quadratic displacement, } \\
\text { Linear velocity }\end{array}$ & $(P 2-P 1)$ & $\Delta t^{3}$ & $1 / 72$ \\
\hline $\begin{array}{c}\text { Linear displacement } \\
\text { (single-field formulation) }\end{array}$ & $(P 1)$ & $\Delta t$ & $1 / 2$ \\
\hline $\begin{array}{l}\text { Quadratic displacement } \\
\text { (single-field formulation) }\end{array}$ & $(P 2)$ & $\Delta t^{3}$ & $1 / 72$ \\
\hline
\end{tabular}


Table II. Local truncation errors for the Galerkin/least-squares method

\begin{tabular}{ccc}
\hline Interpolation & Error rate & Error coefficient \\
\hline$P 0-P 0$ & $\Delta t$ & $1+\left(\hat{t}_{1}+\hat{\tau}_{2}\right)$ \\
\hline$P 1-P 0$ & $\Delta t$ & $\frac{1}{2}+\hat{t}_{1}$ \\
\hline$P 1-P 1$ & $\Delta t^{3}$ & $\frac{1}{36}+\frac{1}{18}\left(\hat{t}_{1}+\hat{t}_{2}\right)+\frac{\hat{t}_{1}^{2}+\hat{\tau}_{2}^{2}+2 \hat{t}_{1} \hat{t}_{2}\left(\hat{t}_{1}+\hat{\tau}_{2}\right)}{18\left(1+2\left(\hat{t}_{1}+\hat{t}_{2}\right)+4 \hat{\tau}_{1} \hat{t}_{2}\right)}$ \\
\hline$P 2-P 1$ & $\Delta t^{3}$ & $\frac{1}{72}+\frac{\hat{t}_{1}\left(1+3 \hat{t}_{1}\right)}{18\left(1+2 \hat{\tau}_{1}\right)}$ \\
\hline$P 1$ & $\Delta t$ & $\frac{1}{2}+\hat{t}$ \\
\hline$P 2$ & $\Delta t^{3}$ & $\frac{1}{72}+\frac{\hat{t}(1+3 \hat{t})}{18(1+2 \hat{t})}$ \\
\hline
\end{tabular}

Note: $\hat{t}=\tau / \Delta t, \hat{t}_{i}=\tau_{i} / \Delta t$

\subsection{Spectral radii}

Spectral radius plots are useful to observe the dissipative properties of an algorithm over the entire frequency domain. Of particular interest is the spectral radius magnitude at the high end of the frequency spectrum. It is desirable for structural dynamics algorithms to attenuate high frequency response because high frequency behaviour is typically the result of spatial discretization and does not represent physical behaviour of the structure. Ideally, the high frequency behaviour should be annihilated in one time step, i.e.

$$
\rho_{\infty}=\lim _{\mathbf{\Omega} \rightarrow \infty} \rho=0
$$

We refer to this desirable attribute as 'asymptotic annihilation' of the high frequency response. Simultaneously, dissipation should be small in the low frequency regime so that the physical response is not overdamped. Low frequency dissipation is best measured by the algorithmic damping ratio; see Section 5.4 .

Figure 1 depicts spectral radii for the time-discontinuous Galerkin method obtained using the six different interpolations. Also shown are the spectral radii for the Hilber-Hughes-Taylor $\alpha$ method (HHT- $\alpha$ method) and the Houbolt algorithm. The HHT- $\alpha$ method is a good second-order accurate semidiscrete algorithm which attenuates high frequency response. ${ }^{4-44}$ All results were computed using $\alpha=-0.3$. The Houbolt method $^{45}$ is the only three-step linear multistep algorithm that attains asymptotic annihilation. (See Hughes ${ }^{40}$ for a discussion of linear multistep methods for second-order equations.) The spectral radii of the $P 1$ single-field formulation and the $P 1-P 0$ two-field formulation are the same since the displacement-difference stencils are identical; likewise the $P 2$ and $P 2-P 1$ spectral radii are identical. Differences between the first-order and third-order accurate algorithms may be easily observed in the low frequency region. Note the $P 0-P 0$ and $P 1-P 1$ elements possess the asymptotic annihilation property. From an accuracy standpoint, $P 1-P 1$ is preferred since it is a third-order accurate method while $P 0-P 0$ is only firstorder accurate and hence too dissipative in the low frequency region. The $P 1$ and $P 1-P 0$ 


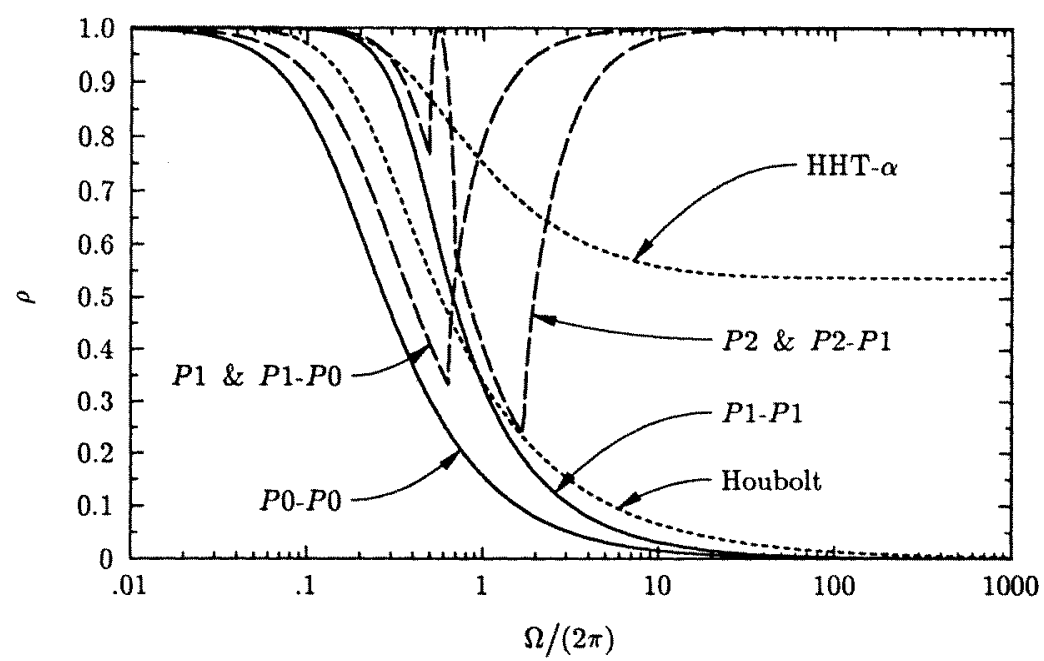

Figure 1. Spectral radii for time-discontinuous Galerkin methods

elements are not useful for transient dynamics since they exhibit first-order accuracy yet do not dampen high frequency response. While the spectral radii for these methods initially decrease, the roots of their characteristic polynomials bifurcate when $\Omega=4 \cdot 0$; the roots remain real-valued for frequencies above this limit. (See Hilber ${ }^{4-43}$ for details about the effects of root bifurcations within the context of semidiscrete algorithms.) The $P 2$ and $P 2-P 1$ elements are third-order accurate but do not dampen the high frequencies due to root bifurcations when $\Omega=3 \cdot 1$ and $\Omega=10 \cdot 8$. Thus, while the time-discontinuous Galerkin method using $P 1-P 1$ elements is an effective algorithm from the perspective of stability and accuracy (its roots do not bifurcate), $P 2$ and $P 2-P 1$ elements need the additional dissipation engendered by the least-squares terms to render viable algorithms for structural dynamics.

Figure 2 depicts the spectral radii of the Galerkin/least-squares method for the six interpolation combinations $\left(\tau=\tau_{1}=\tau_{2}=1 / 2 \Delta t\right)$ and the spectral radii of the HHT $\alpha$ and Houbolt algorithms. All six interpolation combinations exhibit the asymptotic annihilation property. While the roots of $P 1-P 0, P 1, P 2-P 1$ and $P 2$ still bifurcate, the addition of the least-squares terms ensures that their spectral radii are zero in the high frequency limit.

Figure 3 compares the spectral radii of the time-discontinuous Galerkin and Galerkin/leastsquares methods for each of the six interpolation combinations. The influence of the least-squares operators on the spectral radii may be readily observed from these plots.

\subsection{Numerical dissipation and dispersion}

The algorithmic damping associated with the time-discontinuous Galerkin method for the six interpolation combinations is shown in Figure 4 (top figure) as a function of the frequency parameter, $\Omega$. Also included are the algorithmic damping curves for the HHT- $\alpha$ and Houbolt algorithms. The Houbolt algorithm is generally considered to be too dissipative in the low frequency regime; thus, $P 0-P 0, P 1-P 0$ and $P 1$ are clearly too dissipative to be of practical interest. The algorithmic damping curves of $P 1-P 1$ and HHT- $\alpha$ are nearly identical when $\Omega \leqslant 0 \cdot 3 \pi$. It is observed that the $P 2-P 1$ and $P 2$ elements have very little dissipation in the low frequency domain. 

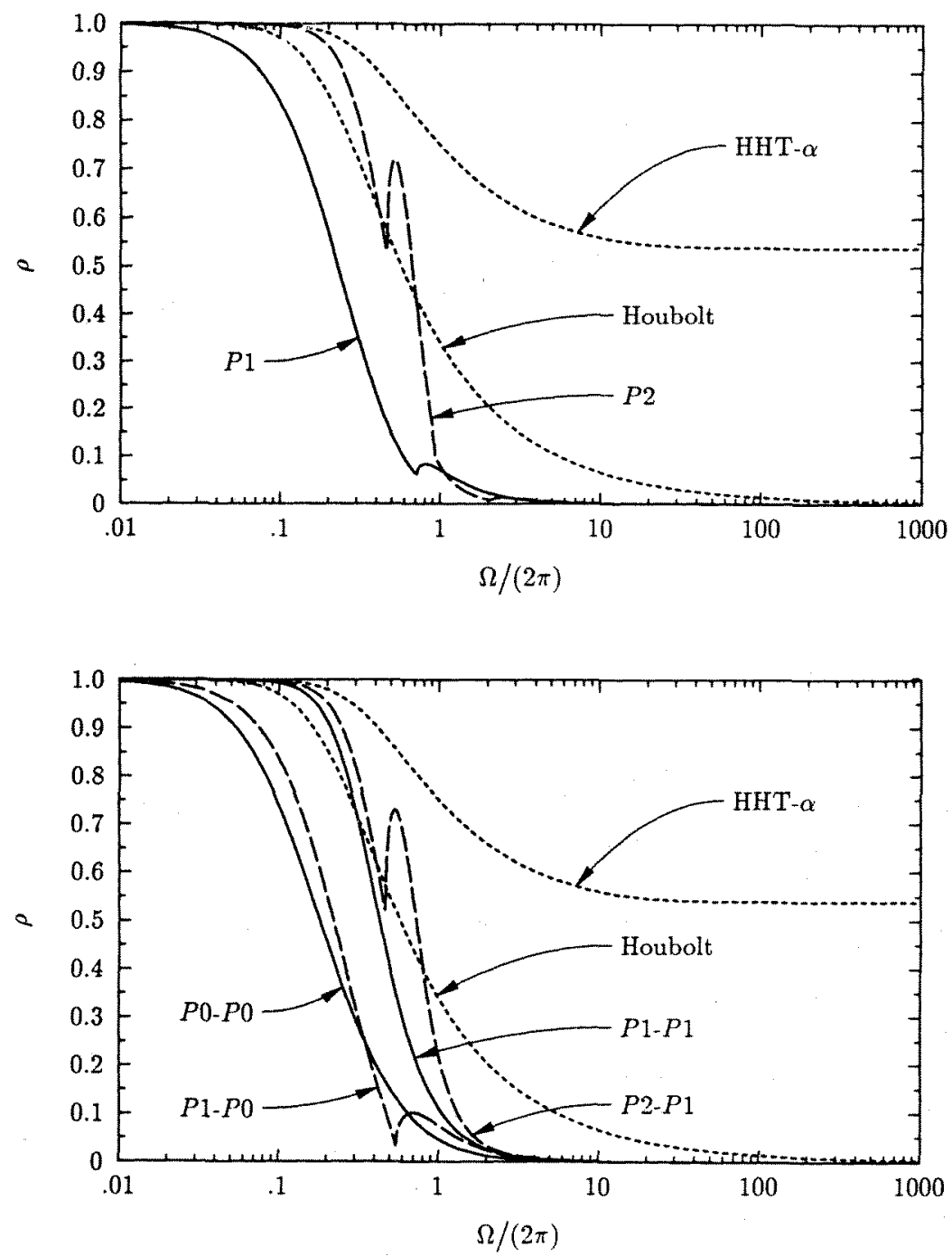

Figure 2. Spectral radii for Galerkin/least-squares methods. (Top: single-field formulation; Bottom: two-field formulation)

Algorithmic damping for the Galerkin/least-squares, HHT- $\alpha$ and Houbolt algorithms is shown in Figure 4 (bottom figure), with $\tau=\tau_{1}=\tau_{2}=1 / 2 \Delta t$. The effect of the least-squares operators is to add dissipation to the time-discontinuous Galerkin formulations; consequently, the algorithmic damping increases. Algorithmic damping curves for $P 0-P 0, P 1-P 0$ and $P 1$ are omitted since these elements are already too dissipative without the least-squares terms. The least-squares terms result in algorithmic damping ratios greater than that of the HHT- $\alpha$ method for all elements. However, when $\Omega<0 \cdot 10 \pi$, there is little difference between the algorithmic damping 
$P 0-P 0$

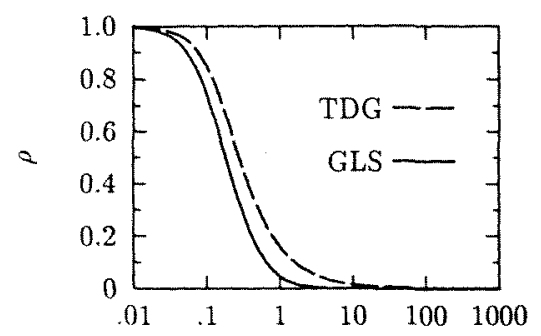

$P 1-P 1$

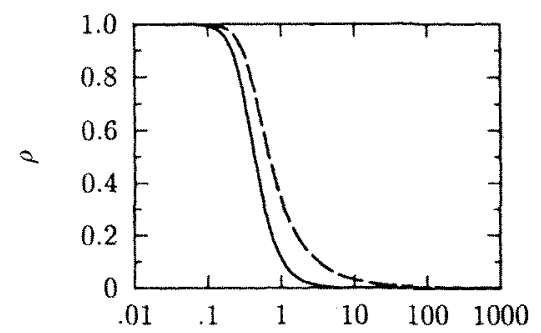

$P 1$

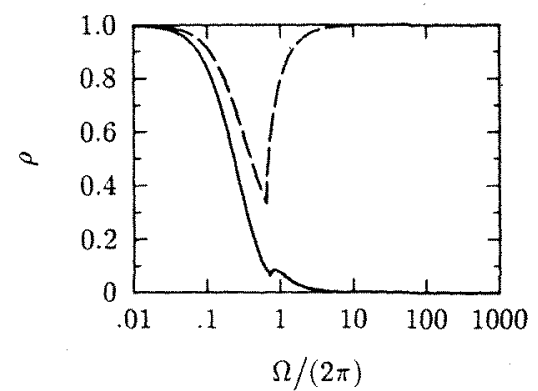

$P 1-P 0$

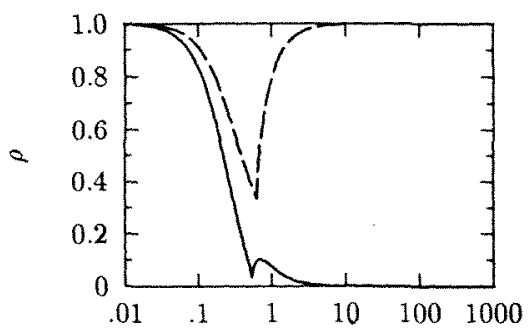

P2-P1

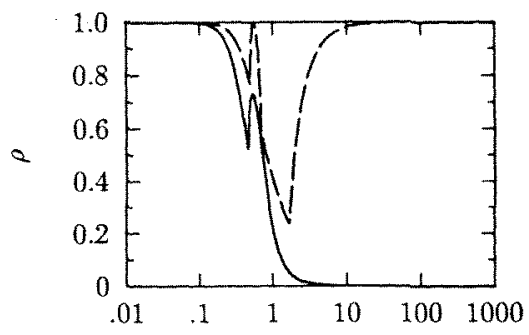

$P 2$

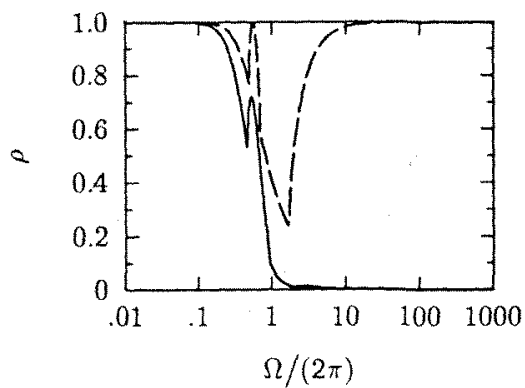

Figure 3. Spectral radii for time-discontinuous Galerkin and Galerkin/least-squares methods. (TDG $\equiv$ time-discontinuous Galerkin method; GLS $\equiv$ Galerkin/least-squares method)

ratios of the HHT- $\alpha$ method and the $P 1-P 1, P 2-P 1$ and $P 2$ elements. It is important to recall that, unlike the HHT- $\alpha$ algorithm, $P 1-P 1, P 2-P 1$ and $P 2$ achieve asymptotic annihilation of high frequency response.

Relative frequency error in the low frequency domain is shown in Figure 5 for the timediscontinuous Galerkin, Galerkin/least-squares, trapezoidal rule, HHT- $\alpha$ and Houbolt algorithms. Dahlquist ${ }^{46}$ proved that, for unconditionally stable linear multistep methods, the trapezoidal rule exhibits the smallest frequency error; thus frequency errors for the HHT- $\alpha$ and 

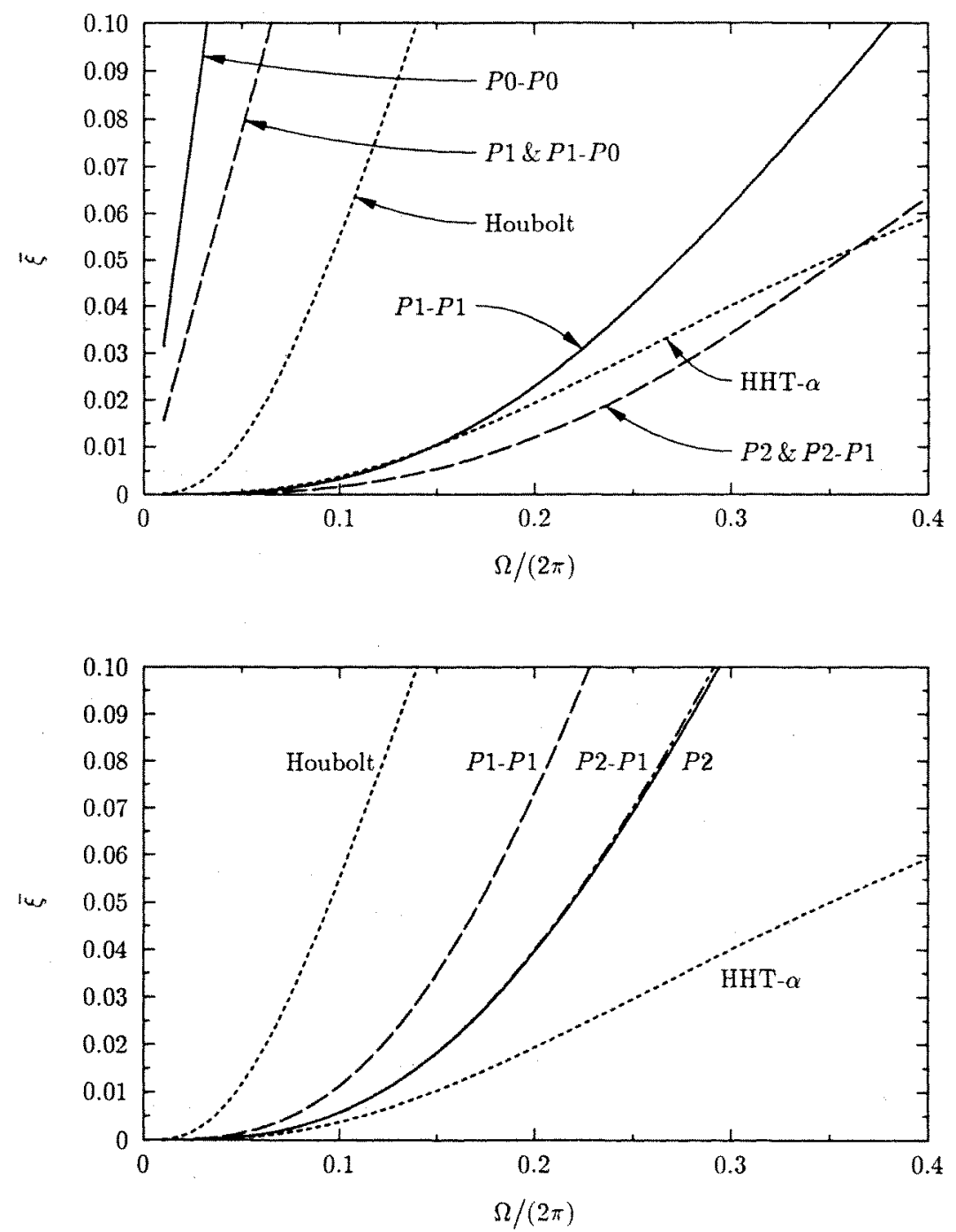

Figure 4. Algorithmic damping ratios for time finite element methods. (Top: time-discontinuous Galerkin method; Bottom: Galerkin/least-squares method)

Houbolt algorithms are greater than that of the trapezoidal rule. Time-discontinuous Galerkin and Galerkin/least-squares formulations do not result in linear multistep algorithms and thus can generate unconditionally stable algorithms which have less numerical dispersion than the trapezoidal rule. The results in Figure 5 demonstrate the superior numerical dispersion characteristics of the $P 1-P 1, P 2-P 1$ and $P 2$ elements. While the frequency errors of $P 0-P 0, P 1-P 0$ and $P 1$ are comparable to those of the semidiscrete formulations, these time finite element methods are not effective time integration schemes owing to poor accuracy and algorithmic damping characteristics. 

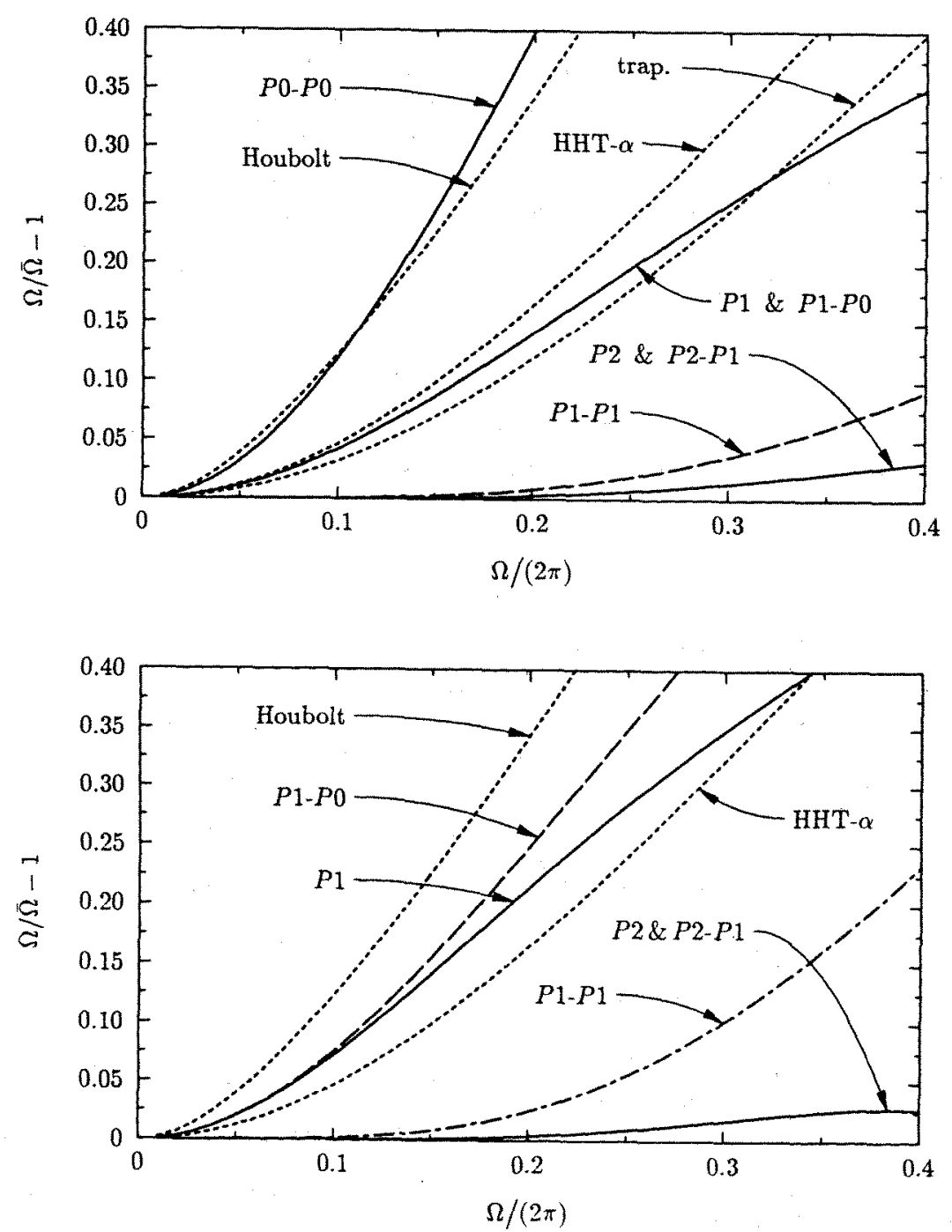

Figure 5. Relative frequency errors for time finite element methods. (Top: time-discontinuous Galerkin method; Bottom: Galerkin/least-squares method)

\section{CONCLUSIONS}

A new time finite element formulation of structural dynamics was presented. The methods are based upon using the time-discontinuous Galerkin method; stabilizing terms having least-squares form are included. Stability and convergence of single-field and two-field formulations were proved. Finite difference analyses of the time finite element methods were performed using various temporal interpolations; the results show the methods possess advantages over commonly used structural dynamics algorithms. In particular, the Galerkin/least-squares formulations achieve high-order accuracy and asymptotically annihilate undesirable high frequency 
response without introducing excessive algorithmic damping in the low frequency regime. In addition, the proposed methods exhibit little numerical dispersion.

Time-discontinuous Galerkin methods typically lead to systems of coupled equations which are larger than those emanating from standard semidiscrete methods. To approach economic competitiveness with existing algorithms, predictor-multicorrector, explicit or implicit-explicit algorithms need to be developed; the challenge is to develop a more economical algorithm that inherits the desirable accuracy and stability properties of the underlying fully coupled methods. This is the subject of a subsequent publication.

\section{ACKNOWLEDGEMENTS}

The research presented herein was conducted by the author at Stanford University; support from the U.S. Office of Naval Research under Contract N00014-88-K-0446 is gratefully acknowledged. The author wishes to thank T. J. R. Hughes for many helpful comments and discussions.

\section{REFERENCES}

1. J. H. Argyris and D. W. Scharpf, 'Finite elements in time and space', Nucl. Eng. Des., 10, 456-464 (1969).

2. G. Bazzi and E. Anderheggen, 'The $\rho$-family of algorithms for time-step integration with improved numerical dissipation', Earthquake eng. struct. dyn., 10, 537-550 (1982).

3. C. Hoff and P. J. Pahl, 'Practical performance of the $\theta_{1}$ method and comparison with other dissipative algorithms in structural dynamics', Comp. Methods Appl. Mech. Eng., 67, 87-110 (1988).

4. C. Hoff and P. J. Pahl, 'Development of an implicit method with numerical dissipation from a generalized single-step algorithm for structural dynamics', Comp. Methods Appl. Mech. Eng., 67, 367-385 (1988).

5. C. Hoff, T. J. R. Hughes, G. Hulbert and P. J. Pahl, 'Extended comparison of the Hilber-Hughes-Taylor $\alpha$-method and the $\theta_{1}$-method', Comp. Methods Appl. Mech. Eng., 76, 87-93 (1989).

6. M. Kawahara and K. Hasegawa, 'Periodic Galerkin finite element method of tidal flow', Int. j. numer. methods eng., 12, $115-127$ (1978).

7. J. Kujawski and C. S. Desai, 'Generalized time finite element algorithm for non-linear dynamic problems', Eng. Computations, 1, 247-251 (1984).

8. O. C. Zienkiewicz and C. J. Parekh, 'Transient field problems--two and three dimensional analysis by isoparametric finite elements', Int. j. numer. methods eng., 2, 61-71 (1970).

9. O. C. Zienkiewicz, The Finite Element Method, McGraw-Hill, London, 1977.

10. O. C. Zienkiewicz, "A new look at the Newmark, Houbolt and other time stepping formulas. A weighted residual approach', Earthquake eng. struct. dyn., 5, 413-418 (1977).

11. O.C. Zienkiewicz, W. L. Wood and R. L. Taylor, 'An alternative single-step algorithm for dynamic problems', Int. $j$. numer. methods eng., 8, 31-40 (1980).

12. O. C. Zienkiewicz, W. L. Wood, N. W. Hine and R. L. Taylor, 'A unified set of single step algorithms', Int. j. numer. methods eng., 20, 1529-1552 (1984).

13. W. H. Reed and T. R. Hill, 'Triangular mesh methods for the neutron transport equation', Report LA-UR-73-479, Los Alamos Scientific Laboratory, Los Alamos, 1973.

14. P. Lesaint and P.-A. Raviart, 'On a finite element method for solving the neutron transport equation', in C. de Boor (ed.), Mathematical Aspects of Finite Elements in Partial Differential Equations, Academic Press, New York, 1974, pp. 89-123.

15. R. Bonnerot and P. Jamet, ' $A$ third order accurate discontinuous finite element method for the one-dimensional Stefan problem', J. Comp. Phys., 32, 145-167 (1979).

16. M. Delfour, W. Hager and F. Trochu, 'Discontinuous Galerkin methods for ordinary differential equations', Math. Comp., 36, 455-473 (1981).

17. T. J. R. Hughes, L. P. Franca and M. Mallet, 'A new finite element formulation for computational fluid dynamics: VI. Convergence analysis of the generalized SUPG formulation for linear time-dependent multidimensional advectivediffusive systems', Comp. Methods Appl. Mech. Eng., 63, 97-112 (1987).

18. T. J. R. Hughes and G. M. Hulbert, 'Space-time finite element methods for elastodynamics: formulations and error estimates', Comp. Methods Appl. Meth. Eng., 66, 339-363 (1988).

19. T. J. R. Hughes, L. P. Franca, G. M. Hulbert, Z. Johan and F. Shakib, 'The Galerkin/least-squares method for advective-diffusive equations', in T. J. R. Hughes and T. E. Tezduyar (eds.), Recent Developments in Computational Fluid Dynamics, AMD Vol. 95, ASME, New York, 1988, pp. 75-99.

20. G. M. Hulbert and T. J. R. Hughes, "Space-time finite element methods for second-order hyperbolic equations', Comp. Methods Appl. Mech. Eng., 84, 327-348 (1990). 
21. J. Jamet, 'Galerkin-type approximations which are discontinuous in time for parabolic equations in a variable domain', SIAM J. Numer. Anal., 15, 912-928 (1978).

22. C. Johnson, U. Nävert and J. Pitkäranta, 'Finite element methods for linear hyperbolic problems', Comp. Methods Appl. Mech. Eng., 45, 285-312 (1984).

23. C. Johnson and J. Pitkäranta, 'An analysis of the discontinuous Galerkin method for a scalar hyperbolic equation', Technical Report MAT-A215, Institute of Mathematics, Helsinki University of Technology, Helsinki, Finland, 1984.

24. C. Johnson, 'Streamline diffusion methods for problems in fluid mechanics', in R. H. Gallagher et al. (eds.), Finite Elements in Fluids - Vol. 6, Wiley, Chichester, U.K., 1986, pp. 251-261.

25. C. Johnson and J. Saranen, 'Streamline diffusion methods for the incompressible Euler and Navier-Stokes equations', Math. Comp., 47, 1-18 (1986).

26. C. Johnson, Numerical Solutions of Partial Differential Equations by the Finite Element Method, Cambridge University Press, Cambridge, 1987.

27. U. Nävert, 'A finite element method for convection-diffusion problems', Ph.D. Thesis, Department of Computer Science, Chalmers University of Technology, Göteborg, Sweden, 1982.

28. F. Shakib, 'Finite element analysis of the compressible Euler and Navier-Stokes equations', Ph.D. Thesis, Division of Applied Mechanics, Stanford University, Stanford, California, 1989.

29. V. Thomée, Galerkin Finite Element Methods for Parabolic Problems, Springer-Verlag, New York, 1984.

30. C. Johnson, 'Error estimates and automatic time step control for numerical methods for stiff ordinary differential equations', Technical Report 1984-27, Department of Mathematics, Chalmers University of Technology and the University of Göteborg, Göteborg, Sweden, 1984.

31. K. Eriksson, C. Johnson and J. Lennblad, 'Optimal error estimates and adaptive time and space step control for linear parabolic problems', Technical Report 1986-06, Department of Mathematics, Chalmers University of Technology and the University of Göteborg, Göteborg, Sweden, 1986.

32. K. Eriksson and C. Johnson, 'Error estimates and automatic time step control for nonlinear parabolic problems, I', SI AM J. Numer. Anal., 24, 12-23 (1987).

33. K. Eriksson and C. Johnson, 'Adaptive finite element methods for parabolic problems: I. A linear model problem', Technical Report 1988-31, Department of Mathematics, Chalmers University of Technology and the University of Göteborg, Göteborg, Sweden, 1988.

34. P. Hansbo, 'Adaptivity and streamline diffusion procedures in the finite element method', Ph.D. Thesis, Department of Structural Mechanics, Chalmers University of Technology, Göteborg, Sweden, 1989.

35. C. Johnson, Y.-Y. Nie and V. Thomée, 'An a posteriori error estimate and automatic time step control for a backward Euler discretization of a parabolic problem', Technical Report 1985-23, Department of Mathematics, Chalmers University of Technology and the University of Göteborg, Göteborg, Sweden, 1985.

36. C. Johnson and A. Szepessy, 'On the convergence of streamline diffusion finite element methods for hyperbolic conservation laws', in T. E. Tezduyar and T. J. R. Hughes (eds.), Numerical Methods for Compressible Flows-Finite Difference, Element and Volume Techniques, AMD Vol. 78, ASME, New York, 1986, pp. 75-91.

37. C. Johnson and A. Szepessy, 'On the convergence of a finite element method for a nonlinear hyperbolic conservation law', Math. Comp., 49, 427-444 (1987).

38. C. Johnson, A. Szepessy and P. Hansbo, 'On the convergence of shock-capturing streamline diffusion finite element methods for hyperbolic conservation laws', Technical Report 1987-21, Department of Mathematics, Chalmers University of Technology and the University of Göteborg, Göteborg, Sweden, 1987.

39. A. Szepessy, 'Convergence of the streamline diffusion finite element method for conservation laws', Ph.D. Thesis, Department of Mathematics, Chalmers University of Technology, Göteborg, Sweden, 1989.

40. T. J. R. Hughes, The Finite Element Method: Linear Static and Dynamic Finite Element Analysis, Prentice-Hall, Englewood Cliffs, New Jersey, 1987.

41. H. M. Hilber, 'Analysis and design of numerical integration methods in structural dynamics', Ph.D. Thesis, Earthquake Engineering Research Center, University of California, Berkeley, 1976.

42. H. M. Hilber, T. J. R. Hughes and R. L. Taylor, 'Improved numerical dissipation for time integration algorithms in structural dynamics', Earthquake eng. struct. dyn., 5, 283-292 (1977).

43. H. M. Hilber and T. J. R. Hughes, "Collocation, dissipation and "overshoot" for time integration schemes in structural dynamics', Earthquake eng. struct. dyn., 6, 99-118 (1978).

44. T. J. R. Hughes, 'Analysis of transient algorithms with particular reference to stability behavior', in T. Belytschko and T. J. R. Hughes (eds.), Computational Methods for Transient Analysis, North-Holland, Amsterdam, 1983, pp. 67-155.

45. J. C. Houbolt, 'A recurrence matrix solution for the dynamic response of elastic aircraft', J. Aeronaut. Sci., 17, 540-550 (1950).

46. G. Dahlquist, 'A special stability problem for linear multistep methods', BIT, 3, 27-43 (1963). 